\title{
Dalton

\section{Improving the photoresponse of copper(I) dyes in dye-sensitized solar cells by tuning ancillary and anchoring ligand modules $t$}

\author{
Biljana Bozic-Weber, Sven Y. Brauchli, Edwin C. Constable, ${ }^{*}$ Sebastian O. Fürer, \\ Catherine E. Housecroft, * Frederik J. Malzner, lain A. Wright and \\ Jennifer A. Zampese
}

\begin{abstract}
The syntheses of five homoleptic copper( $(1)$ complexes $\left[\mathrm{CuL}_{2}\right]\left[\mathrm{PF}_{6}\right]$ are described in which $\mathrm{L}$ is a $4,4^{\prime}$-di(4-bromophenyl)-6,6'-dialkyl-2,2'-bipyridine ligand (compounds 1-4 with methyl, " butyl, isobutyl and hexyl substituents, respectively) or 4,4'-di(4-bromophenyl)-6,6'-diphenyl-2,2'-bipyridine (5). The new ligands 2-5 and copper $(\mathrm{I})$ complexes $\left[\mathrm{CuL}_{2}\right]\left[\mathrm{PF}_{6}\right](\mathrm{L}=\mathbf{1 - 5})$ have been fully characterized. The single crystal structures of $2\left\{\left[\mathrm{Cu}(1)_{2}\right]\left[\mathrm{PF}_{6}\right]\right\} \cdot 3 \mathrm{Me}_{2} \mathrm{CO},\left[\mathrm{Cu}(2)_{2}\right]\left[\mathrm{PF}_{6}\right], 2\left\{\left[\mathrm{Cu}(3)_{2}\right]\left[\mathrm{PF}_{6}\right]\right\} \cdot \mathrm{Et}_{2} \mathrm{O}$ and $\left[\mathrm{Cu}(5)_{2}\right]\left[\mathrm{PF}_{6}\right] \cdot \mathrm{CH}_{2} \mathrm{Cl}_{2}$ have been determined. The first three structures show similar distorted tetrahedral environments for the $\mathrm{Cu}^{+}$ ions with angles between the least squares planes of the bpy domains of 85.6, 86.4 and $82.9^{\circ}$, respectively; in contrast, the $\mathrm{Cu}^{+}$ion in $\left[\mathrm{Cu}(5)_{2}\right]\left[\mathrm{PF}_{6}\right] \cdot \mathrm{CH}_{2} \mathrm{Cl}_{2}$ is in a flattened coordinate environment due to intra-cation face-to-face $\pi$-interactions. The solution absorption spectra of the complexes with ligands 1-4 are virtually identical with an MLCT band with values of $\lambda_{\max }=481-488 \mathrm{~nm}$. In contrast, the absorption spectrum of $\left[\mathrm{Cu}(5)_{2}\right]\left[\mathrm{PF}_{6}\right]$ shows two broad bands in the visible region. Cyclic voltammetric data show that oxidation of the copper(I) centre occurs at a more positive potential in $\left[\mathrm{Cu}(2)_{2}\right]\left[\mathrm{PF}_{6}\right],\left[\mathrm{Cu}(3)_{2}\right]-$ $\left[\mathrm{PF}_{6}\right]$ and $\left[\mathrm{Cu}(4)_{2}\right]\left[\mathrm{PF}_{6}\right]$ than in $\left[\mathrm{Cu}\left(\mathbf{1}_{2}\right]\left[\mathrm{PF}_{6}\right]\right.$ or $\left[\mathrm{Cu}(5)_{2}\right]\left[\mathrm{PF}_{6}\right]$ with the latter being oxidized at the lowest potential. The complexes have been used to prepare dye-sensitized solar cells (DSCS) incorporating heteroleptic dyes of type $\left[\mathrm{Cu}(\mathrm{L})\left(\mathrm{L}_{\text {anchor }}\right)\right]^{+}$where $\mathrm{L}$ is $\mathbf{1 - 5}$ and $\mathrm{L}_{\text {anchor }}$ is a $6,6^{\prime}$-dimethyl-2,2'-bipyridine functionalized in the 4- and 4'-positions with phosphonic acid groups with $\left(L_{\text {anchor }}=7\right)$ and without $\left(L_{\text {anchor }}=6\right)$ a spacer between the metal-binding and anchoring domains. The presence of the spacer results in enhanced performances of the dyes, and the highest energy conversion efficiencies are observed for the dyes $[\mathrm{Cu}(3)(7)]^{+}(\eta=2.43 \%$ compared to $5.96 \%$ for standard dye $\mathrm{N} 719)$ and $[\mathrm{Cu}(5)(7)]^{+}(\eta=2.89 \% \mathrm{com}-$ pared to $5.96 \%$ for N719). Measurements taken periodically over the course of a week indicate that the cells undergo a ripening process (most clearly seen for $[\mathrm{Cu}(5)(6)]^{+}$and $[\mathrm{Cu}(5)(7)]^{+}$) before their optimum performances are achieved. IPCE (EQE) data are presented and confirm that, although the photo-tocurrent conversions are promising (37-49\% for $\lambda_{\max } \approx 480 \mathrm{~nm}$ ), the copper(I) dyes do not realize the broad spectral response exhibited by N719.
\end{abstract}

Received 30th May 2013,

Accepted 28th June 2013

DOI: $10.1039 / \mathrm{c} 3 \mathrm{dt} 51416 \mathrm{a}$

www.rsc.org/dalton based on similarities between the photophysical properties of copper(I) and ruthenium(II) complexes. ${ }^{4,5}$ The design of suitable ligands for copper(I)-based dyes depends critically on information gained from photophysical and theoretical studies. The suitability of oligopyridine copper(I)-based complexes as sensitizers has been confirmed by the results of TD-DFT calculations. ${ }^{6,7}$ In addition to determining orbital composition (in particular, confirming whether the LUMO of a complex possesses contributions from the anchoring units as required for efficient electron injection), DFT and TD-DFT calculations provide insight into the consequences of changes in ligand conformations upon excitation of the complex, ${ }^{8}$ probe energy matching of the HOMO and LUMO of the dye to the 
conduction band of the semiconductor and predict absorption spectra of dyes. ${ }^{9-11}$

Our recent investigations of copper(I)-based dyes have capitalized on the lability of copper(I) bis(diimine) complexes in solution which allows heteroleptic complexes containing $\left\{\mathrm{Cu}^{\mathrm{I}}(\mathrm{bpy})_{2}\right\}$ cores (bpy $=2,2^{\prime}$-bipyridine) to be assembled in situ by facile ligand exchange. ${ }^{9,12-14}$ One of the two ligands in the complex is selected for its ability to anchor the complex to a mesoporous $\mathrm{TiO}_{2}$ surface. Typical choices of anchoring units are carboxylic acid, phosphonic acid or phenol groups, but we have recently shown that DSCs incorporating dyes with phosphonic acid anchoring groups typically exhibit the best performances, ${ }^{9,13,14}$ consistent with the observations of Grätzel. ${ }^{15}$ The ancillary ligand in the dye is designed to provide efficient light-harvesting properties. It is well established that a $6,6^{\prime}$ dimethyl substitution pattern is required in bpy ligands for stabilization of copper(I). ${ }^{16}$ On the other hand, the photophysical and electrochemical properties of copper(I) bis(diimine) complexes are strongly influenced by the electronic and steric effects of the substituents, viz. 6,6'-substituents in bpy and 2,9-substituents in 1,10-phenanthroline (phen). ${ }^{8,17-22}$ Here we investigate the effects of varying the steric demands of the 6,6'-substituents in the bpy derived ancillary ligands 1-4 (Scheme 1) and the effects of going from alkyl to phenyl substituents (5, Scheme 1$)$, in conjunction with the effects of introducing an aromatic spacer in the phosphonic acid anchoring ligands 6 and 7 (Scheme 1). The inclusion of the bromo-substituent in ancillary ligands $\mathbf{1 - 5}$ provides a built-in handle for further derivatization and tuning of electronic properties of the ligand as we have illustrated with the decoration of 1 with first and second generation hole-transport dendrons. ${ }^{14}$ The choice of 6,6'-substituents in 1-4 was made to explore the effects of alkyl chain length and steric demands on the performance of the surface-anchored dye. In anticipation that longer chains might inhibit assembly of dye complexes on the surface, we decided to trial a new anchoring ligand, 7, in which a phenyl spacer extends the spatial separation of the
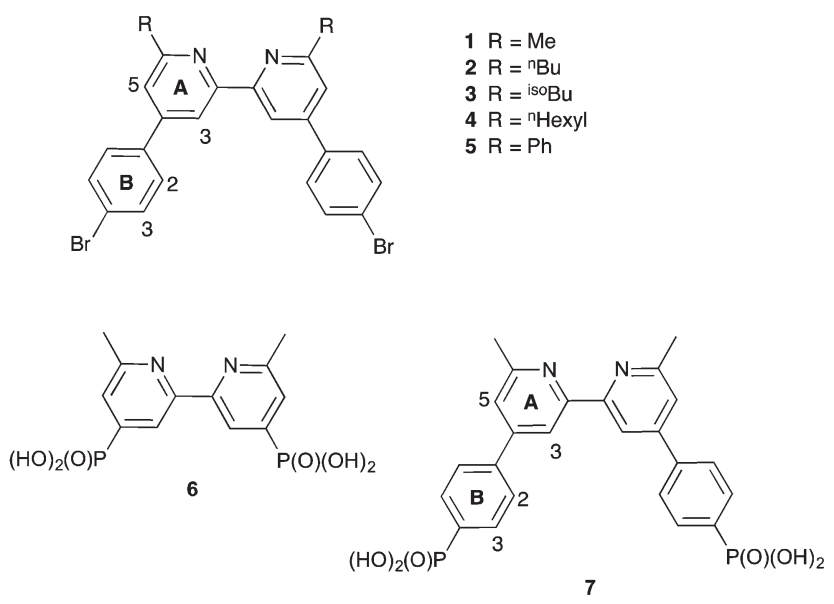

Scheme 1 Ancillary ligands $\mathbf{1 - 5}$ and anchoring ligands $\mathbf{6}$ and 7, with the labelling scheme for ${ }^{1} \mathrm{H}$ NMR spectroscopic assignments. Alkyl chains are labelled $\mathrm{a}, \mathrm{b}$... starting from the ring-attached $\mathrm{CH}_{2}$ unit.

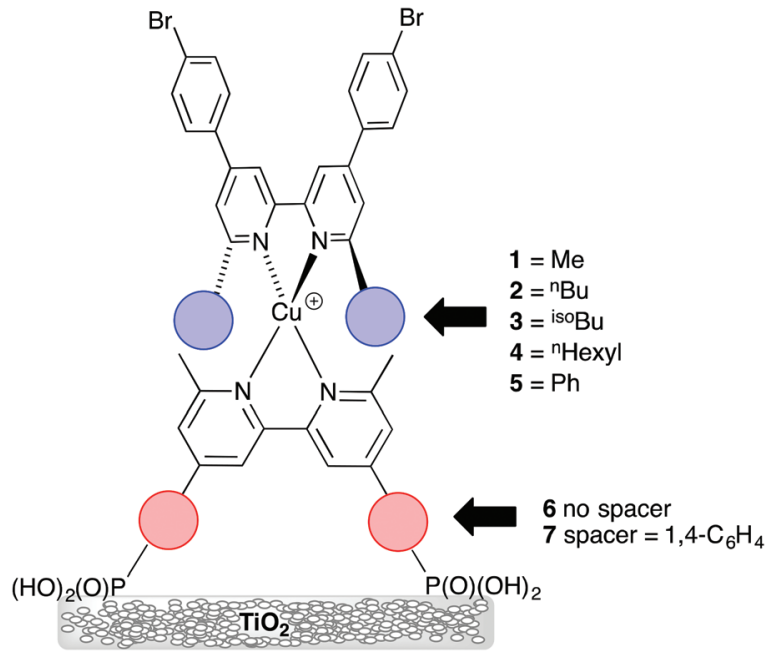

Scheme 2 Schematic representation of the ten dyes studied in this work.

metal centre from the anchoring units compared to dyes incorporating anchoring ligand 6 (Scheme 2).

\section{Experimental}

\section{General}

${ }^{1} \mathrm{H}$ and ${ }^{13} \mathrm{C}$ NMR spectra were recorded using a Bruker Avance III-250, 400 or 500 NMR spectrometer with chemical shifts referenced to residual solvent peaks with respect to $\delta(\mathrm{TMS})=$ 0 ppm. Absorption spectra were recorded on a Cary-5000 spectrophotometer, and FT-IR spectra on a Shimadzu $8400 \mathrm{~S}$ instrument (Golden Gate accessory for solid samples). Electrospray ionization (ESI) mass spectra were recorded on a Bruker Esquire $3000^{\text {plus }}$ instrument.

Electrochemical measurements were made on a $\mathrm{CH}$ Instruments 900B potentiostat using glassy carbon, platinum wire and silver wire as the working, counter, and reference electrodes, respectively. Samples were dissolved in HPLC grade

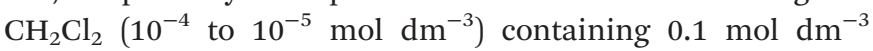
$\left[{ }^{n} \mathrm{Bu}_{4} \mathrm{~N}\right]\left[\mathrm{PF}_{6}\right]$ as the supporting electrolyte; all solutions were degassed with argon. $\mathrm{Cp}_{2} \mathrm{Fe}$ was used as the internal reference.

$(1 E, 5 E)$-1,6-Bis(4-bromophenyl)hexa-1,5-diene-3,4-dione and compound 1 were prepared as previously reported. ${ }^{14}$ Compound $6^{23}$ was prepared by a modified literature method. ${ }^{24}$

\section{1-(2-Oxohexyl)pyridinium iodide}

Iodine (25.6 g, $101 \mathrm{mmol}$ ) was dissolved in pyridine under vigorous stirring (40.8 mL, $39.9 \mathrm{~g}, 505 \mathrm{mmol}$ ) and then hexan-2one $(13 \mathrm{~mL}, 101 \mathrm{mmol})$ was added dropwise over a period of $5 \mathrm{~min}$. The reaction mixture was heated at reflux overnight, and then the solvent was removed under vacuum. The crude material was used without purification for the preparation of compound 2. ${ }^{1} \mathrm{H}$ NMR (400 $\mathrm{MHz}, \mathrm{CDCl}_{3}$ ) $\delta / \mathrm{ppm} 9.17$ (d, $J=$ $\left.5.5 \mathrm{~Hz}, 2 \mathrm{H}, \mathrm{H}^{\mathrm{py} 2}\right), 8.50\left(\mathrm{~m}, 1 \mathrm{H}, \mathrm{H}^{\mathrm{py} 4}\right), 8.06\left(\mathrm{~m}, 2 \mathrm{H}, \mathrm{H}^{\mathrm{py} 3}\right), 6.52$ $\left(\mathrm{s}, 2 \mathrm{H}, \mathrm{H}^{\mathrm{NCH}_{2}}\right), 2.85\left(\mathrm{t}, J=7.4 \mathrm{~Hz}, 2 \mathrm{H}, \mathrm{H}^{\mathrm{CH}_{2}}\right), 1.68(\mathrm{~m}, 2 \mathrm{H}$, $\left.\mathrm{H}^{\mathrm{CH}_{2}}\right), 1.38\left(\mathrm{~m}, 2 \mathrm{H}, \mathrm{H}^{\mathrm{CH}_{2}}\right), 0.93\left(\mathrm{t}, J=7.3 \mathrm{~Hz}, 2 \mathrm{H}, \mathrm{H}^{\mathrm{Me}}\right)$. 


\section{Compound 2}

1-(2-Oxohexyl)pyridinium iodide $(3.00 \mathrm{~g}, 9.83 \mathrm{mmol})$ was dissolved in EtOH $(60 \mathrm{~mL})$ under vigorous stirring. $(1 E, 5 E)-1,6$-Bis(4-bromophenyl)hexa-1,5-diene-3,4-dione (1.03 g, $2.46 \mathrm{mmol}$ ) and $\mathrm{NH}_{4} \mathrm{OAc}(2.84 \mathrm{~g}, 36.9 \mathrm{mmol})$ were added followed by EtOH $(30 \mathrm{~mL})$. The reaction mixture was heated at reflux for 2 days and was then allowed to cool to room temperature while being stirred. The precipitate was collected by filtration, washed with cold EtOH, and then dried under a stream of air. The product was recrystallized twice from EtOH, then from $\mathrm{MeOH}$. Compound 2 was isolated as an off-white solid $(0.630 \mathrm{~g}, 1.09 \mathrm{mmol}$, 44.3\%). m.p. $157{ }^{\circ} \mathrm{C} .{ }^{1} \mathrm{H}$ NMR (500 MHz, $\mathrm{CDCl}_{3}$ ) $\delta / \mathrm{ppm} 8.48$ $\left(\mathrm{d}, J=1.6 \mathrm{~Hz}, 2 \mathrm{H}, \mathrm{H}^{\mathrm{A} 3}\right), 7.63\left(\mathrm{~m}, 8 \mathrm{H}, \mathrm{H}^{\mathrm{B} 2+\mathrm{B} 3}\right), 7.34(\mathrm{~d}, J=$ $\left.1.7 \mathrm{~Hz}, 2 \mathrm{H}, \mathrm{H}^{\mathrm{A} 5}\right), 2.94\left(\mathrm{t}, J=7.8 \mathrm{~Hz}, 4 \mathrm{H}, \mathrm{H}^{\mathrm{a}}\right), 1.83\left(\mathrm{~m}, 4 \mathrm{H}, \mathrm{H}^{\mathrm{b}}\right)$, $1.47\left(\mathrm{~m}, 4 \mathrm{H}, \mathrm{H}^{\mathrm{c}}\right), 0.99\left(\mathrm{t}, J=7.4 \mathrm{~Hz}, 6 \mathrm{H}, \mathrm{H}^{\mathrm{d}}\right) .{ }^{13} \mathrm{C} \mathrm{NMR}$ $\left(126 \mathrm{MHz}, \mathrm{CDCl}_{3}\right) \delta /$ ppm $162.8\left(\mathrm{C}^{\mathrm{A} 6}\right), 156.7\left(\mathrm{C}^{\mathrm{A} 2}\right), 148.3\left(\mathrm{C}^{\mathrm{A} 4}\right)$, $138.1\left(\mathrm{C}^{\mathrm{B} 1}\right), 132.3\left(\mathrm{C}^{\mathrm{B} 2 / 3}\right), 128.9\left(\mathrm{C}^{\mathrm{B} 2 / 3}\right), 123.3\left(\mathrm{C}^{\mathrm{B} 4}\right), 120.4\left(\mathrm{C}^{\mathrm{A} 5}\right)$, 116.6 $\left(\mathrm{C}^{\mathrm{A} 3}\right), 38.7\left(\mathrm{C}^{\mathrm{a}}\right), 32.2\left(\mathrm{C}^{\mathrm{b}}\right), 22.7\left(\mathrm{C}^{\mathrm{c}}\right), 14.2\left(\mathrm{C}^{\mathrm{d}}\right) . \operatorname{IR}\left(\tilde{\nu} / \mathrm{cm}^{-1}\right)$ $2949(\mathrm{w}), 2918(\mathrm{w}), 2869(\mathrm{w}), 2853(\mathrm{w}), 1595(\mathrm{~m}), 1544(\mathrm{~m})$, 1488 (m), 1404 (m), 1274 (m), 1298 (w), 1098 (w), 1008 (m), 821, (s), $758(\mathrm{~m}), 480(\mathrm{~s})$. ESI MS m/z $579.1[\mathrm{M}+\mathrm{H}]^{+}$(calc. 579.1). UV-VIS $\left(\mathrm{CH}_{2} \mathrm{Cl}_{2}, 1.0 \times 10^{-5} \mathrm{~mol} \mathrm{dm}^{-3}\right) \lambda / \mathrm{nm} 256\left(\varepsilon / \mathrm{dm}^{3}\right.$ $\left.\mathrm{mol}^{-1} \mathrm{~cm}^{-1} 43900\right), 302$ (11700). Found: C 61.04, H 5.22, $\mathrm{N} 4.94 ; \mathrm{C}_{30} \mathrm{H}_{30} \mathrm{Br}_{2} \mathrm{~N}_{2} \cdot \frac{1}{2} \mathrm{H}_{2} \mathrm{O}$ requires $\mathrm{C} 61.34, \mathrm{H} 5.32, \mathrm{~N} 4.77 \%$.

\section{1-(4-Methyl-2-oxopentyl)pyridinium iodide}

Iodine $(30.4 \mathrm{~g}, 120 \mathrm{mmol})$ was dissolved in pyridine under vigorous stirring (48.5 mL, $47.4 \mathrm{~g}, 599 \mathrm{mmol}$ ) and then 4-methylpentan-2-one $(15.0 \mathrm{~mL}, 120 \mathrm{mmol})$ was added dropwise over a period of $5 \mathrm{~min}$. The reaction mixture was heated at reflux overnight, after which time it was cooled slowly (over $1 \mathrm{~h}$ ) to room temperature. The solvent was removed under reduced pressure. Diethyl ether was added and the mixture was poured into a flask; the liquid phase was decanted and the solid residue was dissolved in $\mathrm{CHCl}_{3}$. After filtration, the solvent was removed under reduced pressure. The crude material was used without purification for the synthesis of compound $3 .{ }^{1} \mathrm{H}$ NMR $\left(400 \mathrm{MHz}, \mathrm{CDCl}_{3}\right) \delta / \mathrm{ppm} 9.22\left(\mathrm{~d}, J=5.7 \mathrm{~Hz}, 2 \mathrm{H}, \mathrm{H}^{\mathrm{py} 2}\right), 8.58(\mathrm{t}$, $\left.J=7.8 \mathrm{~Hz}, 1 \mathrm{H}, \mathrm{H}^{\mathrm{py} 4}\right), 8.09\left(\mathrm{~m}, 2 \mathrm{H}, \mathrm{H}^{\mathrm{py} 3}\right), 6.38\left(\mathrm{~s}, 2 \mathrm{H}, \mathrm{H}^{\mathrm{NCH}_{2}}\right)$, $2.70\left(\mathrm{~d}, J=6.8 \mathrm{~Hz}, 2 \mathrm{H}, \mathrm{H}^{\mathrm{CH}_{2}}\right), 2.20\left(\mathrm{~m}, 1 \mathrm{H}, \mathrm{H}^{\mathrm{CH}}\right), 0.93(\mathrm{~d}, J=$ $\left.6.7 \mathrm{~Hz}, 6 \mathrm{H}, \mathrm{H}^{\mathrm{Me}}\right)$.

\section{Compound 3}

Compound 3 was prepared in the same manner as 2 starting with 1-(4-methyl-2-oxopentyl)pyridinium iodide $(4.16 \mathrm{~g}$, $14.3 \mathrm{mmol})$ and $(1 E, 5 E)$-1,6-bis(4-bromophenyl)hexa-1,5-diene3,4-dione $(1.50 \mathrm{~g}, 3.57 \mathrm{mmol})$ and $\mathrm{NH}_{4} \mathrm{OAc}(4.13 \mathrm{~g}$, $53.6 \mathrm{mmol})$. The reaction time at reflux was 1 day. Compound 3 was isolated as an off-white solid $(0.401 \mathrm{~g}, 0.693 \mathrm{mmol}$, 19.4\%). m.p. $181{ }^{\circ} \mathrm{C} .{ }^{1} \mathrm{H}$ NMR (500 MHz, $\left.\mathrm{CDCl}_{3}\right) \delta / \mathrm{ppm} 8.48$ $\left(\mathrm{d}, J=1.7 \mathrm{~Hz}, 2 \mathrm{H}, \mathrm{H}^{\mathrm{A} 3}\right), 7.63\left(\mathrm{~m}, 8 \mathrm{H}, \mathrm{H}^{\mathrm{B} 2+\mathrm{B} 3}\right), 7.30(\mathrm{~d}, J=$ $\left.1.7 \mathrm{~Hz}, 2 \mathrm{H}, \mathrm{H}^{\mathrm{A} 5}\right), 2.80\left(\mathrm{~d}, J=7.2 \mathrm{~Hz}, 4 \mathrm{H}, \mathrm{H}^{\mathrm{a}}\right), 2.27\left(\mathrm{~m}, 2 \mathrm{H}, \mathrm{H}^{\mathrm{b}}\right)$, $1.01\left(\mathrm{~d}, J=6.6 \mathrm{~Hz}, 12 \mathrm{H}, \mathrm{H}^{\mathrm{c}}\right) .{ }^{13} \mathrm{C} \mathrm{NMR}\left(126 \mathrm{MHz}, \mathrm{CDCl}_{3}\right)$ $\delta /$ ppm $161.6\left(\mathrm{C}^{\mathrm{A} 6}\right), 156.5\left(\mathrm{C}^{\mathrm{A} 2}\right), 148.1\left(\mathrm{C}^{\mathrm{A} 4}\right), 137.8\left(\mathrm{C}^{\mathrm{B} 1}\right), 132.1$ $\left(\mathrm{C}^{\mathrm{B} 2 / \mathrm{B} 3}\right), 128.8\left(\mathrm{C}^{\mathrm{B} 2 / \mathrm{B} 3}\right), 123.1\left(\mathrm{C}^{\mathrm{B} 4}\right), 121.1\left(\mathrm{C}^{\mathrm{A} 5}\right), 116.5\left(\mathrm{C}^{\mathrm{A} 3}\right)$,
$47.5\left(\mathrm{C}^{\mathrm{a}}\right), 29.0\left(\mathrm{C}^{\mathrm{b}}\right), 22.4\left(\mathrm{C}^{\mathrm{c}}\right) . \mathrm{IR}\left(\tilde{\nu} / \mathrm{cm}^{-1}\right) 3082(\mathrm{w}), 3053(\mathrm{w})$, 2954 (m), 2928 (m), 2913 (w), 2864 (m), 1599 (m), 1543 (m), 1491 (m), 1462 (m), 1376 (m), 1326 (m), 1165 (m), 1010 (s), 896 (m), 828 (s), 814 (s), 765 (s), 744 (m), 658 (m), 590 (m), 526 (s). ESI MS $m / z 579.1[\mathrm{M}+\mathrm{H}]^{+}$(calc. 579.1). UV-VIS $\left(\mathrm{CH}_{2} \mathrm{Cl}_{2}\right.$, $\left.1.0 \times 10^{-5} \mathrm{~mol} \mathrm{dm}^{-3}\right) \lambda / \mathrm{nm} 256\left(\varepsilon / \mathrm{dm}^{3} \mathrm{~mol}^{-1} \mathrm{~cm}^{-1} 58350\right), 304$ (14 360). Found: C 62.03, $\mathrm{H} 5.11, \mathrm{~N} 5.16 ; \mathrm{C}_{30} \mathrm{H}_{30} \mathrm{Br}_{2} \mathrm{~N}_{2}$ requires C 62.30, H 5.23, N 4.84\%.

\section{1-(2-Oxooctyl)pyridinium iodide}

Iodine (16.2 g, $63.9 \mathrm{mmol}$ ) was dissolved in pyridine under vigorous stirring (25.8 mL, $25.2 \mathrm{~g}, 319 \mathrm{mmol})$, and then octan2-one $(10.0 \mathrm{~mL}, 63.9 \mathrm{mmol})$ was added dropwise over a period of $5 \mathrm{~min}$. The reaction mixture was heated to reflux overnight. The reaction mixture was cooled to room temperature over $1 \mathrm{~h}$ and the solvent was removed under reduced pressure. The crude material was used without purification for the synthesis of compound 4. ${ }^{1} \mathrm{H}$ NMR (400 MHz, $\left.\mathrm{CDCl}_{3}\right) \delta / \mathrm{ppm} 9.24(\mathrm{~d}, J=$ $\left.5.8 \mathrm{~Hz}, 2 \mathrm{H}, \mathrm{H}^{\mathrm{py} 2}\right), 8.54\left(\mathrm{~m}, 1 \mathrm{H}, \mathrm{H}^{\mathrm{py} 4}\right), 8.07\left(\mathrm{~m}, 2 \mathrm{H}, \mathrm{H}^{\mathrm{py} 3}\right), 6.47$ $\left(\mathrm{s}, 2 \mathrm{H}, \mathrm{H}^{\mathrm{NCH}_{2}}\right), 2.82\left(\mathrm{t}, J=7.4 \mathrm{~Hz}, 2 \mathrm{H}, \mathrm{H}^{\mathrm{CH}_{2}}\right), 1.65(\mathrm{~m}, 2 \mathrm{H}$, $\mathrm{H}^{\mathrm{CH}_{2}}$ ), 1.27 (overlapping $\left.\mathrm{m}, 6 \mathrm{H}, \mathrm{H}^{\mathrm{CH}_{2}+\mathrm{CH}_{2}+\mathrm{CH}_{2}}\right), 0.85(\mathrm{t}, \mathrm{J}=$ $\left.6.5 \mathrm{~Hz}, 2 \mathrm{H}, \mathrm{H}^{\mathrm{Me}}\right)$.

\section{Compound 4}

Compound $\mathbf{4}$ was prepared in the same manner as 2 starting with 1-(2-oxooctyl)pyridinium iodide $(5.47 \mathrm{~g}, 17.1 \mathrm{mmol})$ and (1E,5E)-1,6-bis(4-bromophenyl)hexa-1,5-diene-3,4-dione $(1.80 \mathrm{~g}$, $4.28 \mathrm{mmol})$ and $\mathrm{NH}_{4} \mathrm{OAc}(4.95 \mathrm{~g}, 64.3 \mathrm{mmol})$. The reaction was heated overnight at reflux. Compound 4 was isolated as an offwhite solid (0.173 g, $0.273 \mathrm{mmol}, 6.36 \%)$. m.p. $135{ }^{\circ} \mathrm{C} .{ }^{1} \mathrm{H}$ NMR (500 MHz, $\mathrm{CDCl}_{3}$ ) $\delta / \mathrm{ppm} 8.48\left(\mathrm{~d}, J=1.7 \mathrm{~Hz}, 2 \mathrm{H}, \mathrm{H}^{\mathrm{A} 3}\right)$, $7.63\left(\mathrm{~m}, 8 \mathrm{H}, \mathrm{H}^{\mathrm{B} 2+\mathrm{B} 3}\right), 7.33\left(\mathrm{~d}, J=1.7 \mathrm{~Hz}, 2 \mathrm{H}, \mathrm{H}^{\mathrm{A} 5}\right), 2.93(\mathrm{t}, J=$ $\left.7.8 \mathrm{~Hz}, 4 \mathrm{H}, \mathrm{H}^{\mathrm{a}}\right), 1.85\left(\mathrm{~m}, 4 \mathrm{H}, \mathrm{H}^{\mathrm{b}}\right), 1.44\left(\mathrm{~m}, 4 \mathrm{H}, \mathrm{H}^{\mathrm{c}}\right), 1.35(\mathrm{~m}$, $\left.8 \mathrm{H}, \mathrm{H}^{\mathrm{d}+\mathrm{e}}\right), 0.99\left(\mathrm{t}, J=7.1 \mathrm{~Hz}, 6 \mathrm{H}, \mathrm{H}^{\mathrm{f}}\right) .{ }^{13} \mathrm{C}$ NMR $(126 \mathrm{MHz}$, $\left.\mathrm{CDCl}_{3}\right) \delta /$ ppm $162.8\left(\mathrm{C}^{\mathrm{A} 6}\right), 156.7\left(\mathrm{C}^{\mathrm{A} 2}\right), 148.3\left(\mathrm{C}^{\mathrm{A} 4}\right), 138.1\left(\mathrm{C}^{\mathrm{B} 1}\right)$, $132.3\left(\mathrm{C}^{\mathrm{B} 2 / \mathrm{B} 3}\right), 128.9\left(\mathrm{C}^{\mathrm{B} 2 / 3}\right), 123.3\left(\mathrm{C}^{\mathrm{B} 4}\right), 120.4\left(\mathrm{C}^{\mathrm{A} 2}\right), 116.6$ $\left(\mathrm{C}^{\mathrm{A} 3}\right), 38.65\left(\mathrm{C}^{\mathrm{a}}\right), 31.92\left(\mathrm{C}^{\mathrm{b}}\right), 29.99\left(\mathrm{C}^{\mathrm{c}}\right), 29.29\left(\mathrm{C}^{\mathrm{d}}\right), 22.80\left(\mathrm{C}^{\mathrm{e}}\right)$, $14.29\left(\mathrm{C}^{\mathrm{f}}\right)$. IR $\left(\tilde{\nu} / \mathrm{cm}^{-1}\right) 2949(\mathrm{~m}), 2924(\mathrm{~m}), 2866(\mathrm{w}), 2853(\mathrm{~m})$, 1597 (m), 1545 (m), 1490 (m), 1462 (m), 1407 (m), 1377 (m), 1069 (m), 1009 (m), 892 (m), 824 (s), 754 (m), 655 (m), 515 (m), $485(\mathrm{~m})$. ESI MS $\mathrm{m} / \mathrm{z} 635.1[\mathrm{M}+\mathrm{H}]^{+}$(calc. 635.1). UV-VIS $\left(\mathrm{CH}_{2} \mathrm{Cl}_{2}, 1.0 \times 10^{-5} \mathrm{~mol} \mathrm{dm}{ }^{-3}\right) \lambda / \mathrm{nm} 255\left(\varepsilon / \mathrm{dm}^{3} \mathrm{~mol}^{-1} \mathrm{~cm}^{-1}\right.$ 49 900), 305 (16 600). Found: $\mathrm{C}$ 62.91, H 5.86, N 4.62; $\mathrm{C}_{34} \mathrm{H}_{38} \mathrm{Br}_{2} \mathrm{~N}_{2} \cdot \mathrm{H}_{2} \mathrm{O}$ requires C 62.58, H 6.18, N 4.29\%.

\section{1-(2-Phenyl-2-oxoethyl)pyridinium iodide}

Iodine $(38.2 \mathrm{~g}, 150 \mathrm{mmol})$ was dissolved in pyridine $(75.0 \mathrm{~mL}$, $73.7 \mathrm{~g}, 932 \mathrm{mmol})$. The solution was stirred until all $\mathrm{I}_{2}$ had dissolved. Then acetophenone $(17.5 \mathrm{~mL}, 18.0 \mathrm{~g}, 150 \mathrm{mmol}, 1.0$ eq.) was added to the solution which was then heated to reflux for $1 \mathrm{~h}$. The heating was stopped and the solution was cooled to $\mathrm{rt}$. The remaining pyridine was removed under vacuum. The solid was washed with ice water and acetone. The product was dried under vacuum and 1-(2-phenyl-2-oxoethyl)pyridinium iodide was isolated as an off-white solid (43.6 g, $134 \mathrm{mmol}$, 90\%). ${ }^{1} \mathrm{H}$ NMR (250 MHz, $\left.\mathrm{CD}_{3} \mathrm{CN}\right) \delta / \mathrm{ppm} 8.77$ (dd, $J=6.6$, 
$\left.1.3 \mathrm{~Hz}, 2 \mathrm{H}, \mathrm{H}^{\mathrm{py} 2}\right), 8.65\left(\mathrm{~m}, 1 \mathrm{H}, \mathrm{H}^{\mathrm{py} 4}\right), 8.17-8.08(\mathrm{~m}, 4 \mathrm{H}$, $\left.\mathrm{H}^{\mathrm{Py} 3+\mathrm{Ph} 2 / 3}\right), 7.78\left(\mathrm{~m}, 1 \mathrm{H}, \mathrm{H}^{\mathrm{Ph} 4}\right), 7.64\left(\mathrm{~m}, 2 \mathrm{H}, \mathrm{H}^{\mathrm{Ph} 2 / 3}\right), 6.4(\mathrm{~s}, 2 \mathrm{H}$, $\mathrm{H}^{\mathrm{NCH}}$ ).

\section{Compound 5}

Compound 5 was prepared in the same manner as 2 starting with 1-(2-phenyl-2-oxoethyl)pyridinium iodide $(1.61 \mathrm{~g}$, $4.95 \mathrm{mmol})$ and $(1 E, 5 E)$-1,6-bis(4-bromophenyl)hexa-1,5-diene3,4-dione (1.04 g, $2.48 \mathrm{mmol})$ and $\mathrm{NH}_{4} \mathrm{OAc}(2.68 \mathrm{~g}$, $37.1 \mathrm{mmol})$. The reaction time at reflux was 1 day. Compound 5 was isolated as an off-white solid $(0.443 \mathrm{~g}, 0.718 \mathrm{mmol}$, 29.0\%). m.p. $318{ }^{\circ} \mathrm{C} .{ }^{1} \mathrm{H}$ NMR (500 MHz, $\mathrm{CD}_{2} \mathrm{Cl}_{2}$ ) $\delta / \mathrm{ppm} 8.91$ $\left(\mathrm{d}, J=1.5 \mathrm{~Hz}, 2 \mathrm{H}, \mathrm{H}^{\mathrm{A} 3}\right), 8.23\left(\mathrm{~m}, 4 \mathrm{H}, \mathrm{H}^{\mathrm{C} 2}\right), 8.04(\mathrm{~d}, J=1.6 \mathrm{~Hz}$, $\left.2 \mathrm{H}, \mathrm{H}^{\mathrm{A} 5}\right), 7.81\left(\mathrm{~m}, 4 \mathrm{H}, \mathrm{H}^{\mathrm{B} 2}\right), 7.72\left(\mathrm{~m}, 4 \mathrm{H}, \mathrm{H}^{\mathrm{B} 3}\right), 7.57(\mathrm{~m}, 4 \mathrm{H}$, $\left.\mathrm{H}^{\mathrm{C} 3}\right), 7.52\left(\mathrm{~m}, 2 \mathrm{H}, \mathrm{H}^{\mathrm{C} 4}\right) .{ }^{13} \mathrm{C}$ NMR $\left(126 \mathrm{MHz}, \mathrm{CD}_{2} \mathrm{Cl}_{2}\right) \delta / \mathrm{ppm}$ $157.6\left(\mathrm{C}^{\mathrm{A} 6}\right), 155.6\left(\mathrm{C}^{\mathrm{A} 2}\right), 150.1\left(\mathrm{C}^{\mathrm{A} 4}\right), 138.8\left(\mathrm{C}^{\mathrm{C} 1}\right), 137.9\left(\mathrm{C}^{\mathrm{B} 1}\right)$, $132.7\left(\mathrm{C}^{\mathrm{B} 3}\right), 129.8\left(\mathrm{C}^{\mathrm{C} 4}\right), 129.5\left(\mathrm{C}^{\mathrm{B} 2}\right), 129.2\left(\mathrm{C}^{\mathrm{C} 3}\right), 127.6\left(\mathrm{C}^{\mathrm{C} 2}\right)$, $124.2\left(\mathrm{C}^{\mathrm{B} 4}\right), 119.5\left(\mathrm{C}^{\mathrm{A} 5}\right), 118.9\left(\mathrm{C}^{\mathrm{A} 3}\right) . \mathrm{IR}\left(\tilde{\nu} / \mathrm{cm}^{-1}\right) 3061(\mathrm{w}), 3035$ (w), 1594 (s), 1571 (m), 1542 (s), 1488 (s), 1405 (m), 1375 (m), 1238 (w), 1181 (w), $1074(\mathrm{~m}), 1025(\mathrm{~m}), 1008(\mathrm{~m}), 884(\mathrm{~m}), 818$ (s), 775 (s), $739(\mathrm{~s}), 690$ (s), $637(\mathrm{~m}), 557$ (m), $538(\mathrm{~m}), 476(\mathrm{~m})$. ESI MS $m / z 619.1[\mathrm{M}+\mathrm{H}]^{+}$(calc. 619.0). UV-VIS $\left(\mathrm{CH}_{2} \mathrm{Cl}_{2}, 1.0 \times\right.$ $\left.10^{-5} \mathrm{~mol} \mathrm{dm}{ }^{-3}\right) \lambda / \mathrm{nm} 265\left(\varepsilon / \mathrm{dm}^{3} \mathrm{~mol}^{-1} \mathrm{~cm}^{-1} 79400\right), 321$ (16 400). Found: $\mathrm{C}$ 64.86, $\mathrm{H} 3.67, \mathrm{~N} 4.54 ; \mathrm{C}_{34} \mathrm{H}_{22} \mathrm{Br}_{2} \mathrm{~N}_{2} \cdot \frac{1}{2} \mathrm{H}_{2} \mathrm{O}$ requires $\mathrm{C} 65.09, \mathrm{H} 3.70, \mathrm{~N} 4.47 \%$.

\section{Compound 7a}

Compound 1 (1.00 g, $2.02 \mathrm{mmol}), \operatorname{Pd}\left(\mathrm{PPh}_{3}\right)_{4}$ (213 mg, $0.18 \mathrm{mmol})$ and $\mathrm{Cs}_{2} \mathrm{CO}_{3}(1.44 \mathrm{~g}, 4.41 \mathrm{mmol})$ were combined in anhydrous THF $(15 \mathrm{~mL})$ in a 10 or $20 \mathrm{~mL}$ microwave vial equipped with a stirrer bar under argon. Diethylphosphite (1.12 $\mathrm{g}, 8.09 \mathrm{mmol}$ ) was added by syringe before the vial was sealed and the reaction mixture heated to $110^{\circ} \mathrm{C}$ for $90 \mathrm{~min}$. The reaction mixture was filtered to give a yellow solution and then the solvent was evaporated under reduced pressure. The resulting brown residue was dissolved in $\mathrm{CH}_{2} \mathrm{Cl}_{2}(20 \mathrm{~mL})$ and stirred with decolourising charcoal for 30 minutes then filtered over celite prior to removal of the solvent under reduced pressure to produce an oily bright yellow residue. The residue was dissolved in acetone $(5 \mathrm{~mL})$ and filtered through a short silica plug eluting with acetone $(25 \mathrm{~mL})$ to give a pale yellow solution. The solvent volume was reduced to $\approx 5 \mathrm{~mL}$ and a mixture of pentane and hexane $(1: 1)$ was added until the solution became turbid. It was heated until clear and then allowed to cool, resulting in the precipitation of $7 \mathbf{a}$ as a white powder (802 mg, 65\%). Single crystals were grown by diffusion of pentane-hexane $(1: 1)$ into a concentrated acetone solution of 7a. m.p. $174{ }^{\circ} \mathrm{C} .{ }^{1} \mathrm{H}$ NMR $\left(500 \mathrm{MHz}, \mathrm{CD}_{3} \mathrm{CN}\right) \delta / \mathrm{ppm} 8.57$ (d, $J=$ $1.2 \mathrm{~Hz}, 2 \mathrm{H}, \mathrm{H}^{\mathrm{A} 3}$ ), 7.92 (overlapping $\left.\mathrm{m}, 8 \mathrm{H}, \mathrm{H}^{\mathrm{B} 2+\mathrm{B} 3}\right), 7.59(\mathrm{~d}, J=$ $\left.1.4 \mathrm{~Hz}, 2 \mathrm{H}, \mathrm{H}^{\mathrm{A} 5}\right), 4.10\left(\mathrm{~m}, 8 \mathrm{H}, \mathrm{H}^{\mathrm{Et}(\mathrm{CH} 2)}\right), 2.68\left(\mathrm{~s}, 6 \mathrm{H}, \mathrm{H}^{\mathrm{Me}}\right), 1.31$ $\left(\mathrm{t}, J=7.0 \mathrm{~Hz}, 12 \mathrm{H}, \mathrm{H}^{\mathrm{Et}(\mathrm{CH} 3)}\right) .{ }^{13} \mathrm{C} \mathrm{NMR}\left(126 \mathrm{MHz}, \mathrm{CD}_{3} \mathrm{CN}\right)$ $\delta /$ ppm $159.9\left(\mathrm{C}^{\mathrm{A} 6}\right), 157.0\left(\mathrm{C}^{\mathrm{A} 2}\right), 149.0\left(\mathrm{C}^{\mathrm{A} 4}\right), 143.2\left(\mathrm{C}^{\mathrm{B} 1}\right), 133.2$ $\left(\mathrm{d}, J_{\mathrm{PC}}=10.1 \mathrm{~Hz}, \mathrm{C}^{\mathrm{B} 2 / \mathrm{B} 3}\right), 130.5\left(\mathrm{~d}, J_{\mathrm{PC}}=187.7 \mathrm{~Hz}, \mathrm{C}^{\mathrm{B} 4}\right), 128.2$ $\left(\mathrm{d}, J_{\mathrm{PC}}=15.0 \mathrm{~Hz}, \mathrm{C}^{\mathrm{B} 2 / \mathrm{B} 3}\right), 122.3\left(\mathrm{C}^{\mathrm{A} 5}\right), 116.8\left(\mathrm{C}^{\mathrm{A} 3}\right), 63.0\left(\mathrm{~d}, J_{\mathrm{PC}}=\right.$ $\left.5.3 \mathrm{~Hz}, \mathrm{C}^{\mathrm{Et}(\mathrm{CH} 2)}\right), 24.7\left(\mathrm{C}^{\mathrm{Me}}\right), 16.6\left(\mathrm{~d}, J_{\mathrm{PC}}=6.3 \mathrm{~Hz}, \mathrm{C}^{\mathrm{Et}(\mathrm{CH} 3)}\right) . \mathrm{IR}$ $\left(\tilde{\nu} / \mathrm{cm}^{-1}\right) 2980(\mathrm{w}), 2936(\mathrm{w}), 2905(\mathrm{w}), 1700(\mathrm{w}), 1591(\mathrm{~m}), 1570(\mathrm{w})$,
1539 (m), 1447 (w), 1382 (m), 1364 (w), 1254 (m), 1242 (s), 1214 (w), 1136 (w), 1121 (m), 1113 (w), 1053 (m), 1023 (s), 1017 (s), $955(\mathrm{~s}), 898(\mathrm{w}), 890(\mathrm{w}), 830(\mathrm{~s}), 798(\mathrm{~m}), 781(\mathrm{~s}), 747(\mathrm{~m})$, $681(\mathrm{~m}), 603(\mathrm{~m}), 571(\mathrm{~s})$. ESI MS m/z 631.2 [M + Na $]^{+}$(calc. 631.2), $609.3[\mathrm{M}+\mathrm{H}]^{+}$(base peak, calc. 609.2). Found $\mathrm{C}$ 63.81, $\mathrm{H}$ 6.34, $\mathrm{N}$ 4.84; $\mathrm{C}_{32} \mathrm{H}_{38} \mathrm{~N}_{2} \mathrm{O}_{6} \mathrm{P}_{2}$ requires $\mathrm{C}$ 63.15, $\mathrm{H}$ 6.29, N $4.60 \%$.

\section{Compound 7}

Compound $7 \mathbf{a}(200 \mathrm{mg}, 0.33 \mathrm{mmol}$ ) was dissolved in aqueous $\mathrm{HCl}(6 \mathrm{M}, 20 \mathrm{~mL})$ and the solution was heated at reflux for $48 \mathrm{~h}$. The solvent was removed under reduced pressure to leave a pale yellow residue. The residue was treated with $\mathrm{AcOH}$ $(15 \mathrm{~mL})$ at reflux in the presence of 5 drops of concentrated aqueous $\mathrm{HCl}$ prior to addition of water. This resulted in the precipitation of 7 as a white powder (163 mg, >99\%). m.p. $>300{ }^{\circ} \mathrm{C}$. ${ }^{1} \mathrm{H}$ NMR $\left.(500 \mathrm{MHz}, \text { DMSO-d })_{6}\right) \delta / \mathrm{ppm} 8.53(\mathrm{~s}, 2 \mathrm{H}$, $\left.\mathrm{H}^{\mathrm{A} 3}\right), 7.95\left(\mathrm{dd}, J_{\mathrm{PH}}=3.1 \mathrm{~Hz}, J_{\mathrm{HH}}=8.4 \mathrm{~Hz}, 2 \mathrm{H}, \mathrm{H}^{\mathrm{B} 2}\right), 8.15(\mathrm{dd}$, $\left.J_{\mathrm{PH}}=12.5 \mathrm{~Hz}, J_{\mathrm{HH}}=8.2 \mathrm{~Hz}, 2 \mathrm{H}, \mathrm{H}^{\mathrm{B} 3}\right), 7.73\left(\mathrm{~s}, 2 \mathrm{H}, \mathrm{H}^{\mathrm{A} 5}\right), 2.68(\mathrm{~s}$, $\left.6 \mathrm{H}, \mathrm{H}^{\mathrm{Me}}\right) .{ }^{13} \mathrm{C}$ NMR (126 MHz, DMSO-d 6 ) $\delta / \mathrm{ppm} 158.7\left(\mathrm{C}^{\mathrm{A} 6}\right)$, $140.0\left(\mathrm{C}^{\mathrm{B} 1}\right), 135.9\left(\mathrm{C}^{\mathrm{A} 4 / \mathrm{B} 4}\right), 134.4\left(\mathrm{C}^{\mathrm{A} 4 / \mathrm{B} 4}\right), 131.3\left(\mathrm{C}^{\mathrm{B} 3}\right), 126.5$ $\left(\mathrm{C}^{\mathrm{B} 2}\right), 121.2\left(\mathrm{C}^{\mathrm{A} 5}\right), 115.2\left(\mathrm{C}^{\mathrm{A} 3}\right), 24.1\left(\mathrm{C}^{\mathrm{Me}}\right), \mathrm{C}^{\mathrm{A} 2}$ not resolved. IR (solid, $\nu / \mathrm{cm}^{-1}$ ) $3244(\mathrm{w}), 3063(\mathrm{w}), 2717$ (br, w), 1645 (m), 1627 (m), 1597 (s), 1544 (w), 1454 (w), 1385 (m), 1360 (w), 1312 (w), 1276 (w), 1238 (m), 1164 (m), 1146 (s), 1137 (s), 1089 (w), 1041 (w), 931 (s), 913 (s), 880 (s), 826 (vs), 761 (m), 728 (w), 680 (vs), 564 (s). Found C 55.06, $\mathrm{H} 4.96, \mathrm{~N} 5.46 ; \mathrm{C}_{24} \mathrm{H}_{22} \mathrm{~N}_{2} \mathrm{O}_{6} \mathrm{P}_{2} \cdot 1.5 \mathrm{H}_{2} \mathrm{O}$ requires $\mathrm{C} 55.07, \mathrm{H}$ 4.81, N 5.35\%. Protonated 7, see text: ${ }^{1} \mathrm{H}$ NMR (500 MHz, $\left.\mathrm{CF}_{3} \mathrm{CO}_{2} \mathrm{D}\right) \delta / \mathrm{ppm} 8.67\left(\mathrm{~s}, 2 \mathrm{H}, \mathrm{H}^{\mathrm{A} 3}\right), 8.33(\mathrm{~s}, 2 \mathrm{H}$, $\left.\mathrm{H}^{\mathrm{A} 5}\right), 8.15\left(\mathrm{dd}, J_{\mathrm{PH}}=14.0 \mathrm{~Hz}, J_{\mathrm{HH}}=8.0 \mathrm{~Hz}, 2 \mathrm{H}, \mathrm{H}^{\mathrm{B} 3}\right), 8.00(\mathrm{dd}$, $\left.J_{\mathrm{PH}}=2.7 \mathrm{~Hz}, J_{\mathrm{HH}}=7.6 \mathrm{~Hz}, 2 \mathrm{H}, \mathrm{H}^{\mathrm{B} 2}\right), 3.04\left(\mathrm{~s}, 6 \mathrm{H}, \mathrm{H}^{\mathrm{Me}}\right)$. ${ }^{13} \mathrm{C}$ NMR (126 MHz, $\left.\mathrm{CF}_{3} \mathrm{CO}_{2} \mathrm{D}\right) \delta / \mathrm{ppm} 161.6\left(\mathrm{C}^{\mathrm{A} 4}\right), 160.8\left(\mathrm{C}^{\mathrm{A} 6}\right)$, $144.2\left(\mathrm{C}^{\mathrm{A} 2}\right), 140.2\left(\mathrm{~d}, J_{\mathrm{PC}}=3.2 \mathrm{~Hz}, \mathrm{C}^{\mathrm{B} 1}\right), 135.2\left(\mathrm{~d}, J_{\mathrm{PC}}=11.4 \mathrm{~Hz}\right.$, $\left.\mathrm{C}^{\mathrm{B} 3}\right), 134.3\left(\mathrm{~d}, J_{\mathrm{PC}}=187 \mathrm{~Hz}, \mathrm{C}^{\mathrm{B} 4}\right), 130.6\left(\mathrm{~d}, J_{\mathrm{PC}}=16.2 \mathrm{~Hz}, \mathrm{C}^{\mathrm{B} 2}\right)$, $130.2\left(\mathrm{C}^{\mathrm{A} 5}\right), 126.7\left(\mathrm{C}^{\mathrm{A} 3}\right), 21.2\left(\mathrm{C}^{\mathrm{Me}}\right)$.

\section{$\left[\mathrm{CuL}_{2}\right]\left[\mathrm{PF}_{6}\right]$ : general procedure}

In a typical reaction, $\left[\mathrm{Cu}(\mathrm{NCMe})_{4}\right]\left[\mathrm{PF}_{6}\right]$ was dissolved in a mixture of $\mathrm{MeCN}$ and $\mathrm{CH}_{2} \mathrm{Cl}_{2}$ and ligand was added. The solution turned deep red immediately. It was stirred overnight, after which time the volume of solvent was reduced in vacuo and the product was precipitated by addition of $\mathrm{Et}_{2} \mathrm{O}$. The solid was redissolved in $\mathrm{MeCN}$ and precipitated again by addition of $\mathrm{Et}_{2} \mathrm{O}$. The dark red product was collected by filtration and was washed with cold $\mathrm{Et}_{2} \mathrm{O}$ and dried in a stream of air.

\section{$\left[\mathrm{Cu}(1)_{2}\right]\left[\mathrm{PF}_{6}\right]$}

$\left[\mathrm{Cu}(\mathrm{NCMe})_{4}\right]\left[\mathrm{PF}_{6}\right](85.0 \mathrm{mg}, 0.228 \mathrm{mmol})$ was reacted with 1 (225 mg, $0.456 \mathrm{mmol}$ ) in $\mathrm{MeCN}(4 \mathrm{~mL})$ and $\mathrm{CH}_{2} \mathrm{Cl}_{2}(16 \mathrm{~mL})$. $\left[\mathrm{Cu}(\mathbf{1})_{2}\right]\left[\mathrm{PF}_{6}\right]$ was isolated as a dark red solid $(217 \mathrm{mg}$, $0.181 \mathrm{mmol}, 79.6 \%)$. Decomp. > $320{ }^{\circ} \mathrm{C} .{ }^{1} \mathrm{H}$ NMR $(500 \mathrm{MHz}$, $\left.\mathrm{CD}_{3} \mathrm{CN}\right) \delta /$ ppm $8.66\left(\mathrm{br}, 4 \mathrm{H}, \mathrm{H}^{\mathrm{A} 3}\right), 7.88\left(\mathrm{~d}, J=7.9 \mathrm{~Hz}, 8 \mathrm{H}, \mathrm{H}^{\mathrm{B} 2 / \mathrm{B} 3}\right)$, $7.83\left(\mathrm{br}, 4 \mathrm{H}, \mathrm{H}^{\mathrm{A} 5}\right), 7.78\left(\mathrm{~d}, J=7.7 \mathrm{~Hz}, 8 \mathrm{H}, \mathrm{H}^{\mathrm{B} 2 / \mathrm{B} 3}\right), 2.37$ (s, $\left.12 \mathrm{H}, \mathrm{H}^{\mathrm{a}}\right) .{ }^{13} \mathrm{C} \mathrm{NMR}\left(126 \mathrm{MHz}, \mathrm{CD}_{3} \mathrm{CN}\right) \delta / \mathrm{ppm} 165.1\left(\mathrm{C}^{\mathrm{A} 6}\right)$, $153.7\left(\mathrm{C}^{\mathrm{A} 2}\right), 149.8\left(\mathrm{C}^{\mathrm{A} 4}\right), 137.1\left(\mathrm{C}^{\mathrm{B} 1}\right), 133.3\left(\mathrm{C}^{\mathrm{B} 2 / \mathrm{B} 3}\right), 130.2$ 
$\left(\mathrm{C}^{\mathrm{B} 2 / \mathrm{B} 3}\right), 124.5\left(\mathrm{C}^{\mathrm{B} 4}\right), 124.8\left(\mathrm{C}^{\mathrm{A} 5}\right), 118.7\left(\mathrm{C}^{\mathrm{A} 3}\right), 25.1\left(\mathrm{C}^{\mathrm{a}}\right)$. ESI MS $m / z$ $1050.8\left[\mathrm{M}-\mathrm{PF}_{6}\right]^{+}$(calc. 1050.9), 495.0 $[1+\mathrm{H}]^{+}$(calc. 495.0). UV-VIS $\left(\mathrm{CH}_{2} \mathrm{Cl}_{2}, 1.0 \times 10^{-5} \mathrm{~mol} \mathrm{dm}{ }^{-3}\right) \lambda / \mathrm{nm}_{280}\left(\varepsilon / \mathrm{dm}^{3} \mathrm{~mol}^{-1}\right.$ $\mathrm{cm}^{-1} 82$ 600), 324 sh (42 200), 488 (11300). Found: C 47.55, $\mathrm{H} 3.16, \mathrm{~N} 4.93 ; \mathrm{C}_{48} \mathrm{H}_{36} \mathrm{Br}_{4} \mathrm{CuF}_{6} \mathrm{~N}_{4} \mathrm{P} \cdot \mathrm{H}_{2} \mathrm{O}$ requires $\mathrm{C} 47.45, \mathrm{H} 3.15$, $\mathrm{N} 4.61 \%$.

\section{$\left[\mathrm{Cu}(2)_{2}\right]\left[\mathrm{PF}_{6}\right]$}

$\left[\mathrm{Cu}(\mathrm{NCMe})_{4}\right]\left[\mathrm{PF}_{6}\right]$ (36.1 $\left.\mathrm{mg}, 0.097 \mathrm{mmol}\right)$ was reacted with 2 $(112 \mathrm{mg}, 0.194 \mathrm{mmol})$ in $\mathrm{MeCN}(10 \mathrm{~mL})$ and $\mathrm{CH}_{2} \mathrm{Cl}_{2}(10 \mathrm{~mL})$. $\left[\mathrm{Cu}(2)_{2}\right]\left[\mathrm{PF}_{6}\right]$ was isolated as a dark red solid $(50.9 \mathrm{mg}$, $0.037 \mathrm{mmol}, 38.5 \%)$. Decomp. > $308{ }^{\circ} \mathrm{C} .{ }^{1} \mathrm{H}$ NMR $(500 \mathrm{MHz}$, $\mathrm{CD}_{3} \mathrm{CN}$ ) $\delta /$ ppm 8.67 (broadened d, $\left.4 \mathrm{H}, \mathrm{H}^{\mathrm{A} 3}\right), 7.89(\mathrm{~d}, J=8.6 \mathrm{~Hz}$, $\left.8 \mathrm{H}, \mathrm{H}^{\mathrm{B} 2}\right), 7.84\left(\mathrm{~d}, J=1.5 \mathrm{~Hz}, 4 \mathrm{H}, \mathrm{H}^{\mathrm{A} 5}\right), 7.78\left(\mathrm{~d}, J=8.6 \mathrm{~Hz}, \mathrm{H}^{\mathrm{B} 3}\right)$, $2.70\left(\mathrm{br}, 8 \mathrm{H}, \mathrm{H}^{\mathrm{a}}\right), 1.40\left(\mathrm{br}, 8 \mathrm{H}, \mathrm{H}^{\mathrm{b}}\right), 0.88\left(\mathrm{br}, 8 \mathrm{H}, \mathrm{H}^{\mathrm{c}}\right), 0.44(\mathrm{br}$, $\left.12 \mathrm{H}, \mathrm{H}^{\mathrm{d}}\right) .{ }^{13} \mathrm{C}$ NMR $\left(126 \mathrm{MHz}, \mathrm{CD}_{3} \mathrm{CN}\right) \delta / \mathrm{ppm} 163.0\left(\mathrm{C}^{\mathrm{A} 6}\right)$, 153.6 $\left(\mathrm{C}^{\mathrm{A} 2}\right), 150.1\left(\mathrm{C}^{\mathrm{A} 4}\right), 137.1\left(\mathrm{C}^{\mathrm{B} 1}\right), 133.4\left(\mathrm{C}^{\mathrm{B} 3}\right), 130.3\left(\mathrm{C}^{\mathrm{B} 2}\right)$, 125.0 $\left(\mathrm{C}^{\mathrm{B} 4}\right), 124.1\left(\mathrm{C}^{\mathrm{A} 5}\right), 119.1\left(\mathrm{C}^{\mathrm{A} 3}\right), 40.3\left(\mathrm{C}^{\mathrm{a}}\right), 32.6\left(\mathrm{C}^{\mathrm{b}}\right), 23.3$ $\left(\mathrm{C}^{\mathrm{c}}\right), 13.7\left(\mathrm{C}^{\mathrm{d}}\right)$. ESI MS $m / z$ 1219.0 $\left[\mathrm{M}-\mathrm{PF}_{6}\right]^{+}$(calc. 1219.1), $579.1[2+\mathrm{H}]^{+}$(calc. 579.1). UV-VIS $\left(\mathrm{CH}_{2} \mathrm{Cl}_{2}, 1.0 \times 10^{-5} \mathrm{~mol}\right.$ $\left.\mathrm{dm}^{-3}\right) \lambda / \mathrm{nm}: 281\left(\varepsilon / \mathrm{dm}^{3} \mathrm{~mol}^{-1} \mathrm{~cm}^{-1} 101500\right), 325 \mathrm{sh}$ (48 000), 486 (13 500). Found: C 52.08, $\mathrm{H} 4.53, \mathrm{~N} 4.29 ; \mathrm{C}_{60} \mathrm{H}_{60} \mathrm{Br}_{4} \mathrm{CuF}_{6} \mathrm{~N}_{4}-\mathrm{P}$. $\mathrm{H}_{2} \mathrm{O}$ requires C 52.10, $\mathrm{H} 4.52, \mathrm{~N} 4.05 \%$.

\section{$\left[\mathrm{Cu}(3)_{2}\right]\left[\mathrm{PF}_{6}\right]$}

$\left[\mathrm{Cu}(\mathrm{NCMe})_{4}\right]\left[\mathrm{PF}_{6}\right](58.7 \mathrm{mg}, 0.158 \mathrm{mmol})$ was reacted with 3 (182 mg, $0.315 \mathrm{mmol})$ in $\mathrm{MeCN}(10 \mathrm{~mL})$ and $\mathrm{CH}_{2} \mathrm{Cl}_{2}(10 \mathrm{~mL})$. $\left[\mathrm{Cu}(3)_{2}\right]\left[\mathrm{PF}_{6}\right]$ was isolated as a dark red solid (186 mg, $0.136 \mathrm{mmol}, 86.4 \%)$. m.p. $338{ }^{\circ} \mathrm{C} .{ }^{1} \mathrm{H}$ NMR (500 MHz, DMSO$\left.\mathrm{d}_{6}\right) \delta /$ ppm $9.08\left(\mathrm{~d}, J=1.4 \mathrm{~Hz}, 4 \mathrm{H}, \mathrm{H}^{\mathrm{A} 3}\right), 8.08\left(\mathrm{~m}, 8 \mathrm{H}, \mathrm{H}^{\mathrm{B} 2}\right), 8.03$ $\left(\mathrm{d}, J=1.4 \mathrm{~Hz}, 4 \mathrm{H}, \mathrm{H}^{\mathrm{A} 5}\right), 7.85\left(\mathrm{~m}, 8 \mathrm{H}, \mathrm{H}^{\mathrm{B} 3}\right), 2.52\left(\mathrm{~s}, 8 \mathrm{H}, \mathrm{H}^{\mathrm{a}}\right)$, 1.72 (septet, d, $\left.J=6.8 \mathrm{~Hz}, 4 \mathrm{H}, \mathrm{H}^{\mathrm{b}}\right), 0.47\left(\mathrm{~d}, J=6.6 \mathrm{~Hz}, 24 \mathrm{H}, \mathrm{H}^{\mathrm{c}}\right)$. ${ }^{13} \mathrm{C}$ NMR (126 MHz, DMSO-d 6 ) $\delta / \mathrm{ppm} 160.6\left(\mathrm{C}^{\mathrm{A} 6}\right), 153.3\left(\mathrm{C}^{\mathrm{A} 2}\right)$, $148.7\left(\mathrm{C}^{\mathrm{A} 4}\right), 135.9\left(\mathrm{C}^{\mathrm{B} 1}\right), 132.7\left(\mathrm{C}^{\mathrm{B} 3}\right), 130.2\left(\mathrm{C}^{\mathrm{B} 2}\right), 124.3\left(\mathrm{C}^{\mathrm{B} 4}\right)$, $123.5\left(\mathrm{C}^{\mathrm{A} 5}\right), 118.1\left(\mathrm{C}^{\mathrm{A} 3}\right), 47.6\left(\mathrm{C}^{\mathrm{a}}\right), 27.5\left(\mathrm{C}^{\mathrm{b}}\right), 21.5\left(\mathrm{C}^{\mathrm{c}}\right)$. ESI MS $\mathrm{m} / \mathrm{z} 1219.1\left[\mathrm{M}-\mathrm{PF}_{6}\right]^{+}$(calc. 1219.1), $579.1[3+\mathrm{H}]^{+}$(calc. 579.1). UV-VIS $\left(\mathrm{CH}_{2} \mathrm{Cl}_{2}, 1.0 \times 10^{-5} \mathrm{~mol} \mathrm{dm}^{-3}\right) \lambda / \mathrm{nm} 282\left(\varepsilon / \mathrm{dm}^{3}\right.$ $\left.\mathrm{mol}^{-1} \mathrm{~cm}^{-1} 98200\right), 323 \mathrm{sh}$ (44 950), 481 (12 100). Found: C 52.51, $\mathrm{H}$ 4.52, $\mathrm{N} 4.38 ; \mathrm{C}_{60} \mathrm{H}_{60} \mathrm{Br}_{4} \mathrm{CuF}_{6} \mathrm{~N}_{4} \mathrm{P}$ requires $\mathrm{C}$ 52.78, $\mathrm{H} 4.43$, N $4.10 \%$.

\section{$\left[\mathrm{Cu}(4)_{2}\right]\left[\mathrm{PF}_{6}\right]$}

$\left[\mathrm{Cu}(\mathrm{NCMe})_{4}\right]\left[\mathrm{PF}_{6}\right](27.4 \mathrm{mg}, 0.074 \mathrm{mmol})$ was reacted with 4 (93.4 mg, $0.147 \mathrm{mmol})$ in $\mathrm{MeCN}(10 \mathrm{~mL})$ and $\mathrm{CH}_{2} \mathrm{Cl}_{2}(10 \mathrm{~mL})$. $\left[\mathrm{Cu}(4)_{2}\right]\left[\mathrm{PF}_{6}\right]$ was isolated as a dark red solid $(40.2 \mathrm{mg}$, $0.027 \mathrm{mmol}, 45.2 \%)$. m.p. $270{ }^{\circ} \mathrm{C} .{ }^{1} \mathrm{H}$ NMR (500 MHz, $\left.\mathrm{CD}_{3} \mathrm{CN}\right)$ $\delta /$ ppm $8.70\left(\mathrm{~s}, 4 \mathrm{H}, \mathrm{H}^{\mathrm{A} 3}\right), 7.90\left(\mathrm{~d}, J=8.5 \mathrm{~Hz}, 8 \mathrm{H}, \mathrm{H}^{\mathrm{B} 2}\right), 7.85(\mathrm{~s}$, $\left.4 \mathrm{H}, \mathrm{H}^{\mathrm{A} 5}\right), 7.79\left(\mathrm{~d}, J=8.5 \mathrm{~Hz}, 8 \mathrm{H}, \mathrm{H}^{\mathrm{B} 3}\right), 2.68\left(\mathrm{~s}, 8 \mathrm{H}, \mathrm{H}^{\mathrm{a}}\right), 1.41(\mathrm{~s}$, $\left.8 \mathrm{H}, \mathrm{H}^{\mathrm{b}}\right), 0.89\left(\mathrm{~s}, 8 \mathrm{H}, \mathrm{H}^{\mathrm{e}}\right), 0.84\left(\mathrm{~s}, 8 \mathrm{H}, \mathrm{H}^{\mathrm{c}}\right), 0.72\left(\mathrm{~s}, 8 \mathrm{H}, \mathrm{H}^{\mathrm{d}}\right), 0.55$ $\left(\mathrm{t}, J=6.6 \mathrm{~Hz}, 12 \mathrm{H}, \mathrm{H}^{\mathrm{f}}\right) .{ }^{13} \mathrm{C} \mathrm{NMR}\left(126 \mathrm{MHz}, \mathrm{CD}_{3} \mathrm{CN}\right) \delta / \mathrm{ppm}$ $163.2\left(\mathrm{C}^{\mathrm{A} 6}\right), 153.6\left(\mathrm{C}^{\mathrm{A} 2}\right), 150.1\left(\mathrm{C}^{\mathrm{A} 4}\right), 137.0\left(\mathrm{C}^{\mathrm{B} 1}\right), 133.3\left(\mathrm{C}^{\mathrm{B} 3}\right)$, $130.2\left(\mathrm{C}^{\mathrm{B} 2}\right), 124.9\left(\mathrm{C}^{\mathrm{B} 4}\right), 124.1\left(\mathrm{C}^{\mathrm{A} 5}\right), 118.9\left(\mathrm{C}^{\mathrm{A} 3}\right), 40.7\left(\mathrm{C}^{\mathrm{a}}\right), 32.0$ $\left(\mathrm{C}^{\mathrm{d}}\right), 30.6\left(\mathrm{C}^{\mathrm{b}}\right), 30.0\left(\mathrm{C}^{\mathrm{c}}\right), 22.9\left(\mathrm{C}^{\mathrm{e}}\right), 14.0\left(\mathrm{C}^{\mathrm{f}}\right)$. ESI MS $\mathrm{m} / z 1331.0$ $\left[\mathrm{M}-\mathrm{PF}_{6}\right]^{+}$(calc. 1331.2), 635.2 [5+ H] $]^{+}$(calc. 635.1). UV-VIS $\left(\mathrm{CH}_{2} \mathrm{Cl}_{2}, 1.0 \times 10^{-5} \mathrm{~mol} \mathrm{dm}{ }^{-3}\right) \lambda / \mathrm{nm} 282\left(\varepsilon / \mathrm{dm}^{3} \mathrm{~mol}^{-1} \mathrm{~cm}^{-1}\right.$ 85 000), 325 sh (41 100), 486 (10 800). Found: C 55.44, H 5.21,
$\mathrm{N}$ 4.18; $\mathrm{C}_{68} \mathrm{H}_{76} \mathrm{Br}_{4} \mathrm{CuF}_{6} \mathrm{~N}_{4} \mathrm{P} \cdot \frac{1}{2} \mathrm{MeCN}$ requires $\mathrm{C}$ 55.32, $\mathrm{H}$ 5.21, N $4.21 \%$.

\section{$\left[\mathrm{Cu}(5)_{2}\right]\left[\mathrm{PF}_{6}\right]$}

$\left[\mathrm{Cu}(\mathrm{NCMe})_{4}\right]\left[\mathrm{PF}_{6}\right]$ (90.4 mg, $\left.0.243 \mathrm{mmol}\right)$ was reacted with 5 (300 mg, $0.485 \mathrm{mmol}$ ) in $\mathrm{MeCN}(5 \mathrm{~mL})$ and $\mathrm{CH}_{2} \mathrm{Cl}_{2}(40 \mathrm{~mL})$. $\left[\mathrm{Cu}(5)_{2}\right]\left[\mathrm{PF}_{6}\right]$ was isolated as a dark red solid $(206 \mathrm{mg}$, $0.143 \mathrm{mmol}, 58.9 \%)$. m.p. $325{ }^{\circ} \mathrm{C} .{ }^{1} \mathrm{H}$ NMR (500 MHz, $\mathrm{CDCl}_{3}$ ) $\delta /$ ppm $8.23\left(\mathrm{~d}, J=1.2 \mathrm{~Hz}, 2 \mathrm{H}, \mathrm{H}^{\mathrm{A} 3}\right.$ ), 7.77 (overlapping $\mathrm{m}, 8 \mathrm{H}$, $\left.\mathrm{H}^{\mathrm{B} 2+\mathrm{B} 3}\right), 7.64\left(\mathrm{~d}, J=1.2 \mathrm{~Hz}, 2 \mathrm{H}, \mathrm{H}^{\mathrm{A} 5}\right), 7.62(\mathrm{~d}, J=7.5 \mathrm{~Hz}, 4 \mathrm{H}$, $\left.\mathrm{H}^{\mathrm{C} 2}\right), 7.05\left(\mathrm{t}, J=7.4 \mathrm{~Hz}, 2 \mathrm{H}, \mathrm{H}^{\mathrm{C} 4}\right), 6.93\left(\mathrm{t}, J=7.6 \mathrm{~Hz}, \mathrm{H}^{\mathrm{C} 3}\right)$. ${ }^{13} \mathrm{C}$ NMR $\left(126 \mathrm{MHz}, \mathrm{CDCl}_{3}\right) \delta / \mathrm{ppm} 156.9\left(\mathrm{C}^{\mathrm{A} 6}\right), 153.6\left(\mathrm{C}^{\mathrm{A} 2}\right)$, $149.5\left(\mathrm{C}^{\mathrm{B} 1}\right), 137.9\left(\mathrm{C}^{\mathrm{C} 1}\right), 135.6\left(\mathrm{C}^{\mathrm{A} 4}\right), 132.9\left(\mathrm{C}^{\mathrm{B} 2 / 3}\right), 129.5\left(\mathrm{C}^{\mathrm{C} 4}\right)$, $128.9\left(\mathrm{C}^{\mathrm{B} 2 / 3}\right), 127.7\left(\mathrm{C}^{\mathrm{C} 3}\right), 127.6\left(\mathrm{C}^{\mathrm{C} 2}\right), 124.8\left(\mathrm{C}^{\mathrm{B} 4}\right), 122.5\left(\mathrm{C}^{\mathrm{A} 5}\right)$, 119.4 $\left(\mathrm{C}^{\mathrm{A} 3}\right)$. ESI MS $m / z 1299.2\left[\mathrm{M}-\mathrm{PF}_{6}\right]^{+}$(calc. 1399.0), 618.8 $[5+\mathrm{H}]^{+}$(calc. 619.0). UV-VIS $\left(\mathrm{CH}_{2} \mathrm{Cl}_{2}, 1.0 \times 10^{-5} \mathrm{~mol} \mathrm{dm}^{-3}\right)$ $\lambda / \mathrm{nm} 245 \mathrm{sh}\left(\varepsilon / \mathrm{dm}^{3} \mathrm{~mol}^{-1} \mathrm{~cm}^{-1} 57000\right), 268$ (72 400), 289 (76 000), 335 (29 300), 423 (5840), 573 (4350). Found: C 55.02, $\mathrm{H} 3.20, \mathrm{~N} 4.14 ; \mathrm{C}_{68} \mathrm{H}_{44} \mathrm{Br}_{4} \mathrm{CuF}_{6} \mathrm{~N}_{4} \mathrm{P} \cdot 2 \mathrm{H}_{2} \mathrm{O}$ requires $\mathrm{C} 55.14$, H 3.27, N $3.78 \%$.

\section{Crystallography}

Data were collected on a Bruker APEX-II diffractometer with data reduction, solution and refinement using the programs APEX $^{25}$ and SHELXL97 or SHELX-13. ${ }^{26}$ ORTEP-type diagrams and structure analysis used Mercury v. 3.0. ${ }^{27,28}$

\section{$7 \mathbf{a}$}

$\mathrm{C}_{32} \mathrm{H}_{38} \mathrm{~N}_{2} \mathrm{O}_{6} \mathrm{P}_{2}, M=608.58$, colourless plate, triclinic, space group $P \overline{1}, a=7.7810(7), b=7.9273(7), c=14.5543(13) \AA ̊, ~ \alpha=$ 75.197(3), $\beta=88.156(3), \gamma=64.398(3)^{\circ}, U=779.57(12) \AA^{3}, Z=1$, $D_{\mathrm{c}}=1.296 \mathrm{Mg} \mathrm{m}^{-3}, \mu(\mathrm{Cu}-\mathrm{K} \alpha)=1.646 \mathrm{~mm}^{-1}, T=123 \mathrm{~K}$. Total 10179 reflections, 2739 unique, $R_{\text {int }}=0.0401$. Refinement of 2681 reflections (233 parameters) with $I>2 \sigma(I)$ converged at final $R_{1}=0.0391\left(R_{1}\right.$ all data $\left.=0.0395\right), \mathrm{w} R_{2}=0.1074\left(\mathrm{w} R_{2}\right.$ all data $=0.1078)$, gof $=1.058$. CCDC 942072 .

\section{$2\left\{\left[\mathrm{Cu}(1)_{2}\right]\left[\mathrm{PF}_{6}\right]\right\} \cdot 3 \mathrm{Me}_{2} \mathrm{CO}$}

$\mathrm{C}_{105} \mathrm{H}_{90} \mathrm{Br}_{8} \mathrm{Cu}_{2} \mathrm{~F}_{12} \mathrm{~N}_{8} \mathrm{O}_{3} \mathrm{P}_{2}, M=2568.09$, yellow plate, triclinic, space group $P \overline{1}, a=11.7229(6), b=14.2639(8), c=15.9549(8) \AA$, $\alpha=93.364(3), \beta=100.301(3), \gamma=91.589(3)^{\circ}, U=2618.4(2) \AA^{3}$, $Z=1, D_{\mathrm{c}}=1.629 \mathrm{Mg} \mathrm{m}^{-3}, \mu(\mathrm{Cu}-\mathrm{K} \alpha)=4.998 \mathrm{~mm}^{-1}, T=123 \mathrm{~K}$. Total 36736 reflections, 9192 unique, $R_{\text {int }}=0.0380$. Refinement of 7490 reflections (657 parameters) with $I>2 \sigma(I)$ converged at final $R_{1}=0.0575\left(R_{1}\right.$ all data $\left.=0.0703\right), \mathrm{w} R_{2}=0.1655$ $\left(\mathrm{w} R_{2}\right.$ all data $\left.=0.1785\right)$, gof $=1.038$. CCDC 942070 .

\section{$\left[\mathrm{Cu}(2)_{2}\right]\left[\mathrm{PF}_{6}\right]$}

$\mathrm{C}_{60} \mathrm{H}_{60} \mathrm{Br}_{4} \mathrm{CuF}_{6} \mathrm{~N}_{4} \mathrm{P}, M=1365.24$, orange block, monoclinic, space group $P 2_{1} / n, a=17.7430(13), b=16.0855(12), c=$ 20.9134(16) А, $\beta=105.193(3)^{\circ}, U=5760.2(8) \AA^{3}, Z=4, D_{\mathrm{c}}=$ $1.574 \mathrm{Mg} \mathrm{m}^{-3}, \mu(\mathrm{Cu}-\mathrm{K} \alpha)=4.560 \mathrm{~mm}^{-1}, T=123 \mathrm{~K}$. Total 63279 reflections, 10359 unique, $R_{\text {int }}=0.0513$. Refinement of 9481 reflections (747 parameters) with $I>2 \sigma(I)$ converged at final $R_{1}$ $=0.0372\left(R_{1}\right.$ all data $\left.=0.0406\right), \mathrm{w} R_{2}=0.0958\left(\mathrm{w} R_{2}\right.$ all data $=$ 0.0985), gof =1.037. CCDC 942069 . 
$2\left\{\left[\mathrm{Cu}(3)_{2}\right]\left[\mathrm{PF}_{6}\right]\right\} \cdot \mathrm{Et}_{2} \mathrm{O}$

$\mathrm{C}_{124} \mathrm{H}_{130} \mathrm{Br}_{8} \mathrm{Cu}_{2} \mathrm{~F}_{12} \mathrm{~N}_{8} \mathrm{OP}_{2}, M=2804.61$, yellow plate, monoclinic, space group $P 2_{1} / c, a=15.5265(7), \quad b=23.9138(9), \quad c=$ $17.7749(7) \AA, \beta=114.893(2)^{\circ}, U=5986.6(4) \AA^{3}, Z=2, D_{\mathrm{c}}=1.556$ $\mathrm{Mg} \mathrm{m}^{-3}, \mu(\mathrm{Cu}-\mathrm{K} \alpha)=4.410 \mathrm{~mm}^{-1}, T=123 \mathrm{~K}$. Total 46225 reflections, 10612 unique, $R_{\text {int }}=0.0480$. Refinement of 8760 reflections (761 parameters) with $I>2 \sigma(I)$ converged at final $R_{1}=$ $0.0467\left(R_{1}\right.$ all data $\left.=0.0581\right), \mathrm{w} R_{2}=0.1168\left(\mathrm{w} R_{2}\right.$ all data $=$ $0.1244)$, gof $=1.037$. CCDC 942071 .

\section{$\left[\mathrm{Cu}(5)_{2}\right]\left[\mathrm{PF}_{6}\right] \cdot \mathbf{C H}_{2} \mathbf{C l}_{2}$}

$\mathrm{C}_{69} \mathrm{H}_{46} \mathrm{Br}_{4} \mathrm{Cl}_{2} \mathrm{CuF}_{6} \mathrm{~N}_{4} \mathrm{P}, M=1530.12$, yellow plate, orthorhombic, space group Pbcn, $a=7.5560(15), b=28.392(7), c=27.854$ (6) $\AA, U=5976(2) \AA^{3}, Z=4, D_{\mathrm{c}}=1.701 \mathrm{Mg} \mathrm{m}^{-3}, \mu(\mathrm{Cu}-\mathrm{K} \alpha)=$ $5.284 \mathrm{~mm}^{-1}, T=123 \mathrm{~K}$. Total 37392 reflections, 5233 unique, $R_{\text {int }}=0.318$. Refinement of 1637 reflections (395 parameters) with $I>2 \sigma(I)$ converged at final $R_{1}=0.0825\left(R_{1}\right.$ all data $=$ $0.2739), \mathrm{w} R_{2}=0.1280\left(\mathrm{w} R_{2}\right.$ all data $\left.=0.1881\right)$, gof $=0.933$. CCDC 942073.

\section{Solar cell fabrication}

The fabrication of DSCs was based on the method of Grätzel and coworkers. ${ }^{29}$ The $\mathrm{TiO}_{2}$ paste was prepared adapting the published method using a three-roll mill (50 EC, EXAKT, Germany), a sonicator bath and terpineol (CAS: 8000-41-7). Each working electrode was made from an FTO glass plate (Solaronix TCO22-7, $2.2 \mathrm{~mm}$ thickness, sheet resistance $\approx 7 \Omega$ square $^{-1}$ ) which was cleaned by sonicating in Hellmanex ${ }^{\circledR}$ surfactant ( $2 \%$ in milliQ water), and rinsed with milliQ water and EtOH. After surface activation in a $\mathrm{UV}^{-\mathrm{O}_{3}}$ system (Model 256220, Jelight Company Inc.) for $20 \mathrm{~min}$, the FTO plate was immersed in aqueous $\mathrm{TiCl}_{4}\left(40 \mathrm{mmol} \mathrm{dm}^{-3}\right)$ at $70{ }^{\circ} \mathrm{C}$ for $30 \mathrm{~min}$, and then washed with milliQ water and EtOH. The FTO plate was dried and a layer of $\mathrm{TiO}_{2}$ paste was screen printed (90 T, Serilith AG, Switzerland). The printed plate was kept in an EtOH chamber for 3 min to reduce surface irregularities of the printed layer and dried for $6 \mathrm{~min}$ at $125{ }^{\circ} \mathrm{C}$ on a heating plate. The screen printing process was repeated 8 times and then the electrodes were gradually heated at $75{ }^{\circ} \mathrm{C}$ for $30 \mathrm{~min}$, at $135^{\circ} \mathrm{C}$ for $15 \mathrm{~min}$, at $325^{\circ} \mathrm{C}$ for $5 \mathrm{~min}$, at $375^{\circ} \mathrm{C}$ for $5 \mathrm{~min}$, at $450{ }^{\circ} \mathrm{C}$ for $15 \mathrm{~min}$ and at $500{ }^{\circ} \mathrm{C}$ for $15 \mathrm{~min}$. After the final sintering, the thickness of the $\mathrm{TiO}_{2}$ layer was 14-16 $\mu \mathrm{m}$ (measured with a Tencor Alpha-Step 500 profilometer). The annealed $\mathrm{TiO}_{2}$ film was post-treated with $40 \mathrm{mmol} \mathrm{dm}^{-3}$ aqueous $\mathrm{TiCl}_{4}$ solution (see above), rinsed with milliQ water and EtOH and sintered at $500{ }^{\circ} \mathrm{C}$ for $30 \mathrm{~min}$.

The electrodes were cooled to $\mathrm{ca} .80^{\circ} \mathrm{C}$ and immersed in a $1 \mathrm{mM}$ DMSO solution of the anchoring ligand 6 or 7 for $20 \mathrm{~h}$. The colourless electrode was removed from the solution, washed with DMSO and EtOH and dried under a stream of $\mathrm{N}_{2}$. The electrode with the adsorbed anchoring ligand was immersed in a $1 \mathrm{mM}$ MeCN solution of $\left[\mathrm{Cu}(\mathbf{L})_{2}\right]\left[\mathrm{PF}_{6}\right](\mathrm{L}=\mathbf{1 - 4})$ and in a $1 \mathrm{mM} \mathrm{CH} \mathrm{Cl}_{2}$ solution of $\left[\mathrm{Cu}(5)_{2}\right]\left[\mathrm{PF}_{6}\right]$ for 4 days to produce red-orange coloured electrodes. The electrodes were removed from the solution and were washed with $\mathrm{CH}_{2} \mathrm{Cl}_{2}$ and dried under a stream of $\mathrm{N}_{2}$.

Each counter electrode was prepared from an FTO glass plate (Solaronix TCO22-7, $2.2 \mathrm{~mm}$ thickness, sheet resistance $\approx 7 \Omega$ square $^{-1}$ ) with a previously drilled hole. Residual organic impurities were removed by heating for $15 \mathrm{~min}$ at $500{ }^{\circ} \mathrm{C}$ on a heating plate and the perforated plate was washed with water, then $0.1 \mathrm{M} \mathrm{HCl}$ solution in EtOH and finally ultrasonicated in an acetone bath for $15 \mathrm{~min}$. It was dried on a heating plate at $500{ }^{\circ} \mathrm{C}$ for $15 \mathrm{~min}$. The Pt catalyst was deposited on the FTO glass plate by coating with Platisol T $(2 \times 25.0 \mu \mathrm{L})$, Solaronix, and dried on a heating plate at $500{ }^{\circ} \mathrm{C}$ for $15 \mathrm{~min}$.

The dye-covered $\mathrm{TiO}_{2}$ electrode and $\mathrm{Pt}$ counter-electrode were assembled using a thermoplast hot-melt sealing foil (Solaronix, Meltonix 1170-25 Series, 25 microns thick) by heating while pressing them together. The electrolyte comprised LiI $\left(0.1 \mathrm{~mol} \mathrm{dm}^{-3}\right), \mathrm{I}_{2}\left(0.05 \mathrm{~mol} \mathrm{dm}^{-3}\right)$, 1-methylbenzimidazole $\left(0.5 \mathrm{~mol} \mathrm{dm}^{-3}\right)$ and 1-butyl-3-methylimidazolinium iodide $\left(0.6 \mathrm{~mol} \mathrm{dm}^{-3}\right)$ in methoxypropionitrile, and was introduced into the cell by vacuum backfilling. The hole on the counter electrode was finally sealed using the hot-melt sealing foil and a cover glass.

The solar cell measurements and testing protocol were performed using fully masked cells. A black coloured copper sheet was used for masking with a single aperture of an average area of $0.06012 \mathrm{~cm}^{2}$ (with a standard deviation of 1\%) placed over the screen printed dye-sensitized $\mathrm{TiO}_{2}$ circle. The area of the aperture in the mask was smaller than the active area of the dye-sensitized $\mathrm{TiO}_{2}$ dot $\left(0.288 \mathrm{~cm}^{2}\right)$. For complete masking, tape was also applied over the edges and rear of the cell. Current density-voltage $(I-V)$ measurements were made by irradiating from behind using a light source SolarSim 150 (100 $\left.\mathrm{mW} \mathrm{cm}^{-2}=1 \mathrm{sun}\right)$. The power of the simulated light was calibrated by using a reference Si photodiode. The standard dye N719 was purchased from Solaronix.

The quantum efficiency measurements were performed on an Spe-Quest quantum efficiency setup from Rera Systems (Netherlands) equipped with a $100 \mathrm{~W}$ halogen lamp (QTH) and a lambda 300 grating monochromator from Lot Oriel. The monochromatic light was modulated to $3 \mathrm{~Hz}$ using a chopper wheel from ThorLabs. The cell response was amplified with a large dynamic range IV converter from CVI Melles Griot and then measured with an SR830 DSP Lock-In amplifier from Stanford Research.

\section{Results and discussion}

\section{Synthesis and characterization of ancillary ligands}

We have previously described the synthesis of compound 1 using Krönhke methodology ${ }^{30}$ by the treatment of $(1 E, 5 E)$ 1,6-bis(4-bromophenyl)hexa-1,5-diene-3,4-dione with 1-(2-oxopropyl)pyridinium chloride and ammonium acetate. ${ }^{14} \mathrm{Com}$ pounds 2, 3, 4, and 5 were prepared according to Scheme 3 using the appropriate "pyridacylpyridinium salts" to introduce 
<smiles>O=C(/C=C/c1ccc(Br)cc1)C(=O)/C=C/c1ccc(Br)cc1</smiles><smiles>[R]C(=O)ONC(=O)O</smiles><smiles>[R]c1cc(-c2ccc(Br)cc2)cc(-c2cc([R])nc(-c3ccc(Br)cc3)c2)n1</smiles>

Scheme 3 Synthetic route to compounds 2-5

the desired $\mathrm{R}$ groups at the 6,6'-positions of the 2,2'bipyridine.

The electrospray mass spectrum of each of compounds 2-5 exhibited a peak envelope as the base peak which corresponded to $[\mathrm{M}+\mathrm{H}]^{+}$and with an isotope pattern characteristic of the presence of two bromine atoms. The ${ }^{1} \mathrm{H}$ and ${ }^{13} \mathrm{C}$ NMR spectra were in accord with a 4,4',6,6'-substituted 2,2'-bipyridine, and the spectra were assigned using COSY, NOESY, DEPT, HMQC and HMBC methods. The ${ }^{1} \mathrm{H}$ NMR resonances for protons $\mathrm{H}^{\mathrm{A} 5}$ and $\mathrm{H}^{\mathrm{A} 3}$ were distinguished by the appearance of a cross peak in the HMBC spectrum of each of compounds 2-4 between signals for $\mathrm{C}^{\mathrm{a}}$ and $\mathrm{H}^{\mathrm{A} 5}$. In the $500 \mathrm{MHz}{ }^{1} \mathrm{H}$ NMR spectra of 2, 3 and 4, the signals for $\mathrm{H}^{\mathrm{B} 2}$ and $\mathrm{H}^{\mathrm{B} 3}$ overlap, and unambiguous assignments of the $\mathrm{C}^{\mathrm{B} 2}$ and $\mathrm{C}^{\mathrm{B} 3}$ resonances were not made from the HMQC or HMBC spectra.

Compounds 1-4 each contain alkyl substituents in the 6,6'positions and their electronic absorption spectra are similar, being dominated by an intense band at $255 \mathrm{~nm}$ with a weaker band close to $305 \mathrm{~nm}$ (Fig. 1). Introduction of the 6- and 6'-phenyl substituents in compound 5 red-shifts both absorptions (to 265 and $321 \mathrm{~nm}$ ). The enhancement of intensity of

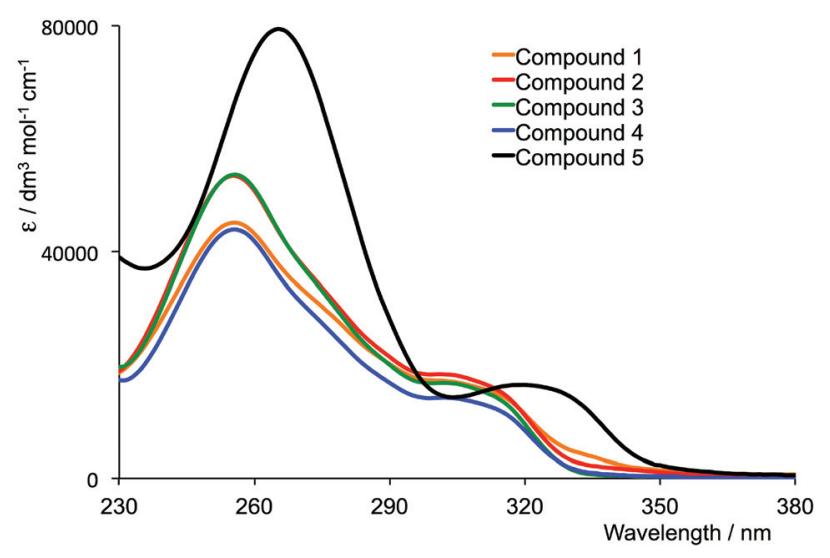

Fig. 1 Absorption spectra of ligands $\mathbf{1 - 5}\left(\mathrm{CH}_{2} \mathrm{Cl}_{2}, 1.0 \times 10^{-5} \mathrm{~mol} \mathrm{dm}{ }^{-3}\right)$. the higher energy band is consistent with the latter arising from $\pi^{*} \leftarrow \pi$ transitions. The lower energy absorption shows no change in intensity along the series of five compounds, and is assigned to $\pi^{*} \leftarrow \mathrm{n}$ (py) transitions.

In terms of ligand design for dyes for DSCs, compound $\mathbf{5}$ appears superior to 1-4 in terms of red-shifting of the spectral response, and we later confirm that this trend is preserved in the homoleptic copper(I) complexes of these ligands.

\section{Synthesis and characterization of anchoring ligand 7}

Compatibility between anchoring and ancillary ligands is of prime importance in our copper-containing heteroleptic dyes. We have previously made extensive use of anchoring ligand $6^{9,13,14}$ and have shown that, even though 6 binds the copper(I) ion close to the $\mathrm{TiO}_{2}$ surface, it remains suited to ancillary ligands with sterically demanding substituents. ${ }^{9}$ On the other hand, we argued that the in situ assembly of copper(I) dyes in which the bpy-derived ancillary ligand contains long chain or aromatic 6,6'-substituents could be enhanced by introducing a spacer between the metal-binding domain of the anchoring ligand and the phosphonic acid anchors. With this in mind, we prepared ligand 7 (Scheme 4) as the first in a series of new phosphonic acid anchoring ligands.

Scheme 4 summarizes the synthesis of 7 from the corresponding ester, 7a (in turn obtained from ligand 1); preparation of the latter was based upon a literature method. ${ }^{31}$ After<smiles>Cc1cc(-c2ccc(Br)cc2)cc(-c2cc(C)nc(-c3ccc(Br)cc3)c2)n1</smiles>

(i)<smiles>CCOPc1ccc(-c2cc(-c3cc(-c4ccc(OCC)cc4)cc(C)n3)cc(C)n2)cc1</smiles><smiles>Cc1cc(-c2ccc([Po]([O-])O[OH2+])cc2)cc(-c2cc(C)nc(-c3ccc([Po]([O-])O[OH2+])cc3)c2)n1</smiles>

Scheme 4 Synthesis of 7: (i) $\left[\mathrm{Pd}\left(\mathrm{PPh}_{3}\right)_{4}\right], \mathrm{Cs}_{2} \mathrm{CO}_{3}, \mathrm{HPO}_{3} \mathrm{Et}_{2}, \mathrm{THF}_{\text {, microwave, }}$ $110^{\circ} \mathrm{C}, 90 \mathrm{~min}$; (ii) aqueous $\mathrm{HCl}$, reflux, $48 \mathrm{~h}$. 


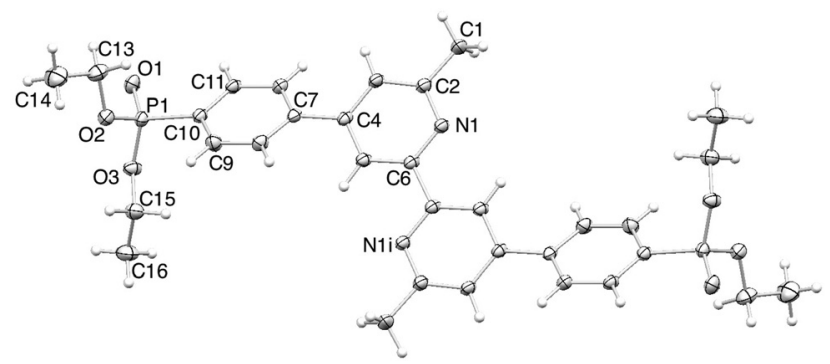

Fig. 2 ORTEP representation of compound $\mathbf{7 a}$ (ellipsoids plotted at $40 \%$ probability level; only the major occupancy sites are shown). Symmetry code $\mathrm{i}=1-$ $x, 1-y, 1-z$. Selected bond distances and angles: N1-C2 $=1.3389(14), N 1-C 6$ $=1.3442(13), \mathrm{C} 10-\mathrm{P} 1=1.7888(9), \mathrm{P} 1-\mathrm{O} 1=1.4629(9), \mathrm{P} 1-\mathrm{O} 3=1.5457(16), \mathrm{P} 1-$ $\mathrm{O} 2=1.5885(8), \mathrm{O} 2-\mathrm{C} 13=1.4515(14), \mathrm{O} 3-\mathrm{C} 15=1.461(3) \AA ; \mathrm{C} 2-\mathrm{N} 1-\mathrm{C} 6=$ 117.68(8), O1-P1-O3, 107.04(8), O1-P1-O2 = 115.27(4), O3-P1-O2 = 103.00 (7), O1-P1-C10 = 113.61(5), O3-P1-C10 = 110.31(6), O2-P1-C10 = 107.07(5) ${ }^{\circ}$

deprotection by treatment with aqueous $\mathrm{HCl}$ and workup, 7a was isolated in $65 \%$ yield. The base peak in the ESI mass spectrum $\left(\mathrm{m} / z\right.$ 609.3) arose from the $[\mathrm{M}+\mathrm{H}]^{+}$ion and a much less intense peak at $m / z 631.2$ was assigned to $[\mathrm{M}+\mathrm{Na}]^{+}$.

$\mathrm{X}$-ray quality crystals of $7 \mathbf{a}$ were grown by diffusion of a mixture of pentane and hexane ( $1: 1$ by volume) into a concentrated acetone solution of the compound. Structural determination confirmed the structure shown in Fig. 2. The bpy unit adopts the expected trans conformation and the two halves of the molecule are related by an inversion centre. Compound 7a is disordered and the OEt group containing $\mathrm{O} 3$ has been modelled over two sites with 49 and 51\% occupancies; the methyl group of the second OEt unit has been modelled over two sites of occupancies 74 and $26 \%$. The phenyl ring is twisted through $29.3^{\circ}$ with respect to the pyridine ring. Although the molecules pack with the aromatic domains over one another, the presence of the methyl substituents prevents efficient faceto-face $\pi$-stacking interactions. The dominant packing forces involve $\mathrm{CH} \cdots \mathrm{O}$ and $\mathrm{CH} \cdots \mathrm{N}$ contacts.

Quantitative conversion of the ester $7 \mathrm{a}$ into phosphonic acid 7 was achieved using aqueous $\mathrm{HCl}(6 \mathrm{M})$ at reflux followed by treatment of the residue obtained with aqueous acetic acid. This latter step appears to be essential for the isolation of pure 7. Compound 7 is poorly soluble in most common solvents and it was not possible to obtain an electrospray mass spectrum. However, satisfactory elemental analysis was obtained for the hydrate $7 \cdot \mathrm{H}_{2} \mathrm{O}$. The solubility of 7 in DMSO- $\mathrm{d}_{6}$ was sufficient to permit a ${ }^{1} \mathrm{H}$ NMR spectrum to be recorded, but ${ }^{13} \mathrm{C}$ NMR resonances could only be assigned using HMQC and HMBC methods; no cross peaks associated with $\mathrm{C}^{\mathrm{A} 2}$ (see Scheme 1 for atom labelling) in the HMBC spectrum were observed. Compound 7 dissolved readily in $\mathrm{CF}_{3} \mathrm{CO}_{2} \mathrm{D}$. The ${ }^{1} \mathrm{H}$ NMR spectrum was consistent with protonation of 7 , although it is difficult to assess from the change in the chemical shift of the signal for $\mathrm{H}^{\mathrm{A} 3}\left(\delta 8.53 \mathrm{ppm}\right.$ for 7 in DMSO- $\mathrm{d}_{6}$ to $\delta 8.67 \mathrm{ppm}$ for the compound in $\mathrm{CF}_{3} \mathrm{CO}_{2} \mathrm{D}$ ) whether the species present is $\left[\mathrm{H}_{2} 7\right]^{2+}$ (trans-conformation of the bpy unit) or $[\mathrm{H} 7]^{+}$(cis-bpy). These conformational changes have been confirmed structurally for salts of $[\mathrm{Hdmbpy}]^{+}$and $\left[\mathrm{H}_{2} \mathrm{dmbpy}\right]^{2+}$ (dmbpy $=6,6^{\prime}-$ dimethyl-2,2'-bipyridine), ${ }^{32}$ and we have recently observed that addition of aliquots of trifluoroacetic acid to a $\mathrm{CDCl}_{3}$ solution of a ligand closely related to 7 leads to an initial downfield shift of the signals for $\mathrm{H}^{\mathrm{A} 3}$ from $\delta 8.39$ to $8.53 \mathrm{ppm}$, followed by an upfield shift to $\delta 8.34 \mathrm{ppm}$ corresponding to formation of first the monoprotonated and then the diprotonated ligand. ${ }^{33}$ Fully assigned NMR spectroscopic data for protonated 7 are given in the Experimental section.

\section{Synthesis and characterization of homoleptic copper(I) complexes}

Reaction of each of ligands 1-5 with $\left[\mathrm{Cu}(\mathrm{NCMe})_{4}\right]\left[\mathrm{PF}_{6}\right]$ in $\mathrm{CH}_{2} \mathrm{Cl}_{2}$ at room temperature resulted in the formation of a red complex characterized as $\left[\mathrm{CuL}_{2}\right]\left[\mathrm{PF}_{6}\right]$. In the electrospray mass spectrum of each complex, the base peak corresponded to $\left[\mathrm{M}-\mathrm{PF}_{6}\right]^{+}$. The combination of copper and bromine isotopes leads to the two most intense peaks in the isotope pattern for the $\left[\mathrm{M}-\mathrm{PF}_{6}\right]^{+}$peak being of almost equal intensity. This is exemplified for $\left[\mathrm{Cu}(3)_{2}\right]\left[\mathrm{PF}_{6}\right]$ in Fig. 3. The lower $\mathrm{m} / \mathrm{z}$ value of each pair is listed in the Experimental section.

The ${ }^{1} \mathrm{H}$ and ${ }^{13} \mathrm{C}$ NMR spectra of the complexes were consistent with a single ligand environment in each complex. The spectra have been fully assigned using routine $2 \mathrm{D}$ methods.

$\mathrm{X}$-ray quality crystals of $2\left\{\left[\mathrm{Cu}(\mathbf{1})_{2}\right]\left[\mathrm{PF}_{6}\right]\right\} \cdot 3 \mathrm{Me}_{2} \mathrm{CO}$ were grown by $\mathrm{Et}_{2} \mathrm{O}$ diffusion into an acetone solution of the complex at room temperature, and Fig. 4 shows the structure of the $\left[\mathrm{Cu}(1)_{2}\right]^{+}$cation. The distorted tetrahedral coordination geometry of atom $\mathrm{Cu} 1$ is less flattened than in $\left[\mathrm{Cu}(\mathrm{dmbpy})_{2}\right]^{+}$ $\left(\right.$ dmbpy $=6,6^{\prime}$-dimethyl-2,2'-bipyridine); in $\left[\mathrm{Cu}(\mathbf{1})_{2}\right]^{+}$, the angle between the least squares planes containing $\mathrm{Cu} 1$ and each bpy unit is $85.6^{\circ}$, compared to angles of $74.3^{\circ}$ in $\left[\mathrm{Cu}(\mathrm{dmbpy})_{2}\right]-$ $\left[\mathrm{PF}_{6}\right],{ }^{9} 80.9^{\circ}$ in $\left[\mathrm{Cu}(\mathrm{dmbpy})_{2}\right]\left[\mathrm{BF}_{4}\right]^{34}$ and $80.9^{\circ}$ in $\left[\mathrm{Cu}(\mathrm{dmbpy})_{2}\right]-$ $\left[\mathrm{ClO}_{4}\right]^{35}$ The flexibility of the $\mathrm{Cu}(\mathrm{bpy})_{2}$-unit is consistent with the suggestion ${ }^{35}$ that packing forces are responsible for the detailed geometry at the copper(I) centre. The two bpy units are close to planar (angles between the planes of the rings containing $\mathrm{N} 1 / \mathrm{N} 2$ and $\mathrm{N} 3 / \mathrm{N} 4=5.5$ and $7.9^{\circ}$, respectively), and the angles between the planes of the pairs of bonded phenyl and pyridine rings range from 10.1 to $34.9^{\circ}$. The variation in the latter is a consequence of packing interactions. Pairs of phenylpyridine units containing $\mathrm{N} 1 / \mathrm{C} 13$ and $\mathrm{N} 1^{i} / \mathrm{C}_{1} 3^{\mathrm{i}}$ (symmetry

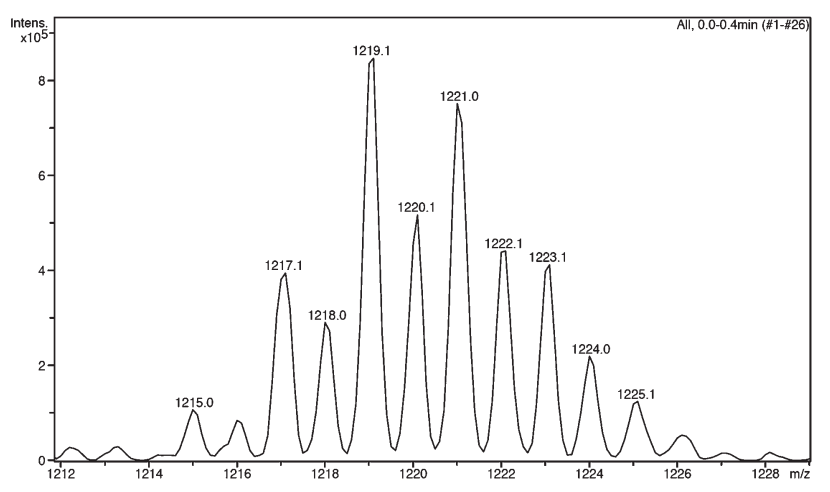

Fig. 3 The base peak envelope in the ESI mass spectrum of $\left[\mathrm{Cu}(3)_{2}\right]\left[\mathrm{PF}_{6}\right]$. 


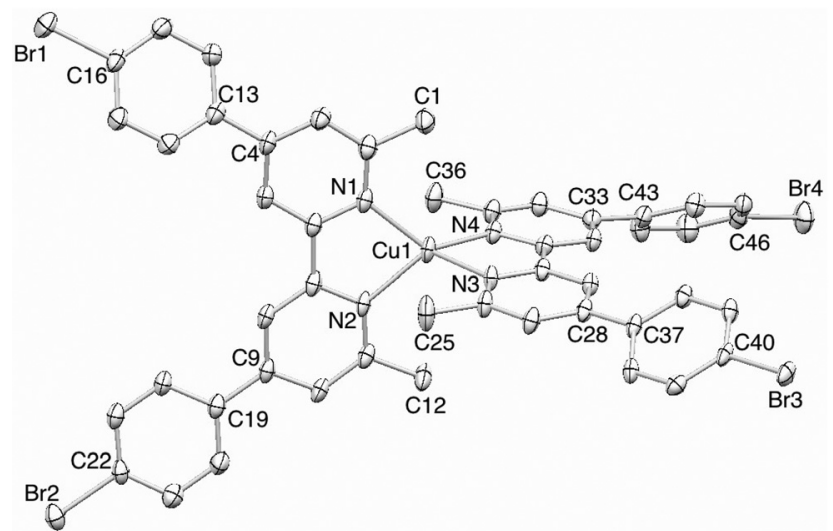

Fig. 4 ORTEP diagram of the $\left[\mathrm{Cu}(\mathbf{1})_{2}\right]^{+}$cation in $2\left\{\left[\mathrm{Cu}(\mathbf{1})_{2}\right]\left[\mathrm{PF}_{6}\right]\right\} \cdot 3 \mathrm{Me}_{2} \mathrm{CO}$ ( $\mathrm{H}$ atoms omitted and ellipsoids plotted at $40 \%$ probability level; only the major occupancy sites are shown). Bond parameters in the coordination sphere: Cu1$\mathrm{N} 4=2.010(4), \mathrm{Cu} 1-\mathrm{N} 1=2.027(4), \mathrm{Cu} 1-\mathrm{N} 3=2.034(4), \mathrm{Cu} 1-\mathrm{N} 2=2.038(4) \AA$; N4-Cu1-N1 = 130.67(18), N4-Cu1-N3 = 80.68(16), N1-Cu1-N3 = 124.04(16), N4-Cu1-N2 = 129.03(16), N1-Cu1-N2 = 81.49(17), N3-Cu1-N2 = 116.14(17) .

code $\mathrm{i}=1-x,-y, 1-z)$ sandwich one acetone solvent molecule which is disordered over two sites positioned about an inversion centre. The phenyl ring of the phenylpyridine unit containing $\mathrm{N} 2$ is $\pi$-stacked over its symmetry related counterpart (symmetry code $=1-x, 1-y, 1-z$ ) at a separation of $3.4 \AA$ (Fig. S1†), and a similar interaction occurs between the phenylpyridine units containing $\mathrm{N} 3$ and $\mathrm{N} 3{ }^{\mathrm{ii}}$ (symmetry code $\mathrm{ii}=3-x, 1-y, 2-z)$. The phenylpyridine unit with N4 shows the greatest deviation from planarity and accommodates a hydrogen-bonded pair of acetone molecules over the pyridine ring $\left(\mathrm{C} 102 \mathrm{H} 10 \mathrm{E} . . . \mathrm{O} 100^{\mathrm{iii}}=1.65 \AA\right.$; symmetry code $\mathrm{iii}=2-x$, $-y, 2-z$ ). The space-filling diagram in Fig. 5a illustrates the shielding of the copper(I) centre by the methyl substituents.

Single crystals of $\left[\mathrm{Cu}(2)_{2}\right]\left[\mathrm{PF}_{6}\right]$ were grown by $\mathrm{Et}_{2} \mathrm{O}$ diffusion into an acetone solution of the complex; the structure of the $\left[\mathrm{Cu}(2)_{2}\right]^{+}$cation is shown in Fig. 6. The distorted tetrahedral coordination geometry of $\mathrm{Cu} 1$ is essentially the same as that in $\left[\mathrm{Cu}(\mathbf{1})_{2}\right]^{+}$with an angle between least squares planes containing $\mathrm{Cu} 1$ and each bpy unit of $86.4^{\circ}$. Each bpy unit is slightly twisted (angles between the planes of the rings containing N1/ $\mathrm{N} 2$ and $\mathrm{N} 3 / \mathrm{N} 4=7.1$ and $12.3^{\circ}$, respectively), and the angles between the planes of the pairs of bonded phenyl and pyridine rings lie in the range $11.1-29.8^{\circ}$, again a consequence of packing interactions. The butyl substituents of the ligand containing N3 and N4 are disordered; each of the three terminal C and attached $\mathrm{H}$ atoms have been modelled over two sites (C31, C32 and C33 with fractional occupancies 0.54 and 0.46 , and C46, C47 and C48 with fractional occupancies 0.51 and 0.49). The presence of the disorder makes it meaningless to comment on the relative orientations of the butyl groups. Nonetheless, using the major occupancy sites, Fig. 5b illustrates how the butyl chains wrap around the copper(I) centre.

Single crystals of $2\left\{\left[\mathrm{Cu}(3)_{2}\right]\left[\mathrm{PF}_{6}\right]\right\} \cdot \mathrm{Et}_{2} \mathrm{O}$ were obtained by $\mathrm{Et}_{2} \mathrm{O}$ diffusion into an acetone-chloroform solution of the complex. Fig. 7 shows the structure of the $\left[\mathrm{Cu}(3)_{2}\right]^{+}$cation. The

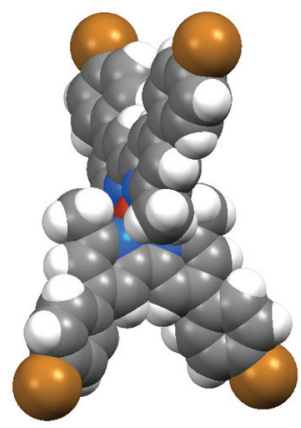

(a)

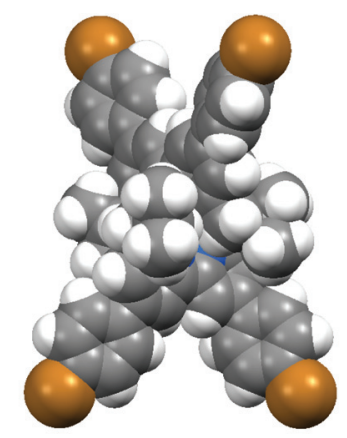

(c)

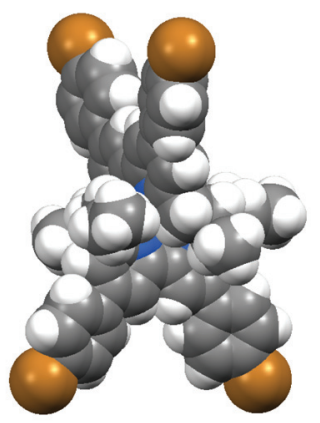

(b)

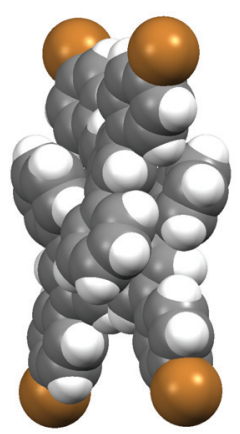

(d)
Fig. 5 Space-filling representation of the (a) $\left[\mathrm{Cu}(\mathbf{1})_{2}\right]^{+}, \quad$ (b) $\left[\mathrm{Cu}(\mathbf{2})_{2}\right]^{+}$, (c) $\left[\mathrm{Cu}(\mathbf{3})_{2}\right]^{+}$and $(\mathrm{d})\left[\mathrm{Cu}(\mathbf{5})_{2}\right]^{+}$cations. In $\left[\mathrm{Cu}(\mathbf{2})_{2}\right]^{+}$, only the major occupancy sites of the two disordered butyl chains are shown.

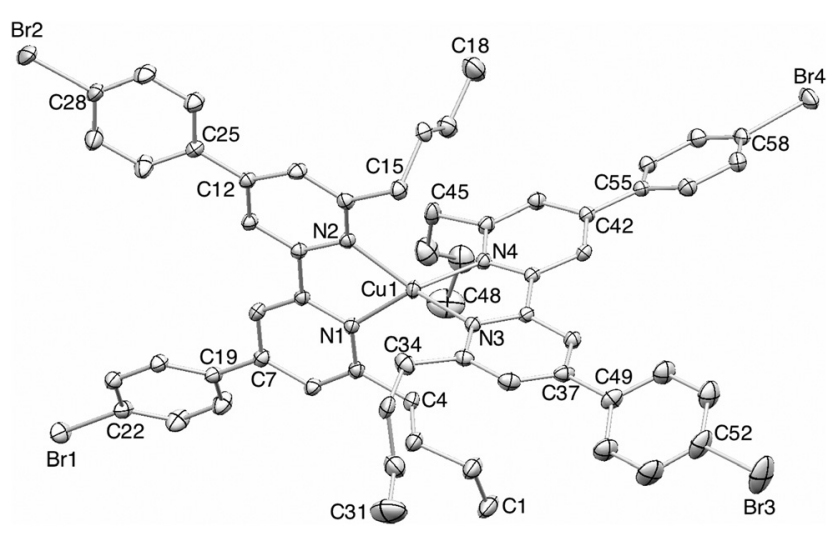

Fig. 6 ORTEP representation of the $\left[\mathrm{Cu}(\mathbf{2})_{2}\right]^{+}$cation in $\left[\mathrm{Cu}(\mathbf{2})_{2}\right]\left[\mathrm{PF}_{6}\right](\mathrm{H}$ atoms omitted and ellipsoids plotted at 40\% probability level; only the major occupancy sites are shown). Bond parameters in the coordination sphere: $\mathrm{Cu} 1-\mathrm{N} 1=$ 2.0368(14), Cu1-N2 = 2.0391(14), Cu1-N3 = 2.0283(15), Cu1-N4 = 2.0485(13) Aं N3-Cu1-N1 = 130.38(6), N3-Cu1-N2 = 126.86(6), N1-Cu1-N2 = 81.35(6), N3-Cu1-N4 = 81.16(6), N1-Cu1-N4 = 117.95(6), N2-Cu1-N4 125.08(5) .

distorted tetrahedral coordination environment is similar to those in $2\left\{\left[\mathrm{Cu}(\mathbf{1})_{2}\right]\left[\mathrm{PF}_{6}\right]\right\} \cdot 3 \mathrm{Me}_{2} \mathrm{CO}$ and $\left[\mathrm{Cu}(2)_{2}\right]\left[\mathrm{PF}_{6}\right]$; the angle between the least squares planes containing $\mathrm{Cu} 1$ and each bpy unit is $82.9^{\circ}$. However, one bpy unit in $\left[\mathrm{Cu}(3)_{2}\right]^{+}$is significantly more twisted than in the complexes containing 1 and 2: angles between the planes of the rings with $\mathrm{N} 1 / \mathrm{N} 2$ and N3/N4 of 12.3 and $29.0^{\circ}$, and this is probably a consequence of steric crowding originating from the four isobutyl groups which 


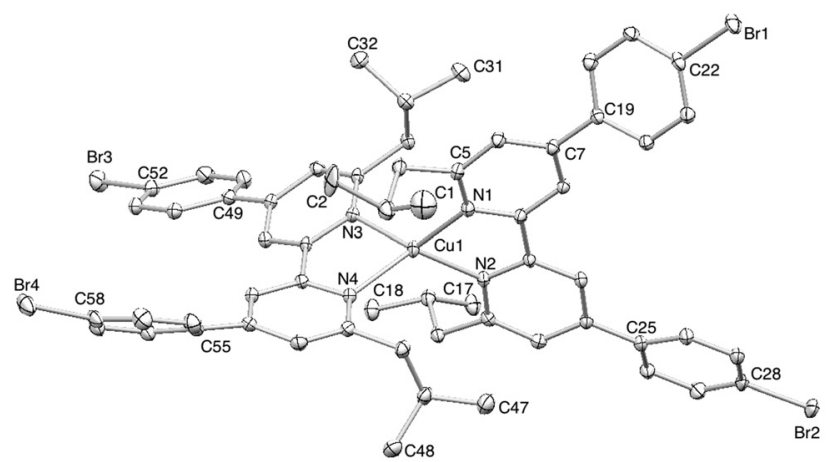

Fig. 7 ORTEP representation of the $\left[\mathrm{Cu}(3)_{2}\right]^{+}$cation in $\left[\mathrm{Cu}(\mathbf{3})_{2}\right]\left[\mathrm{PF}_{6}\right](\mathrm{H}$ atoms omitted and ellipsoids plotted at $40 \%$ probability level). Bond parameters in the coordination sphere: Cu1-N2 $=2.029(3), \mathrm{Cu} 1-\mathrm{N} 4=2.039(3)$, Cu1-N1 $=2.049(3)$, Cu1-N3 = 2.063(3) $\AA$; N2-Cu1-N4 = 124.31(11), N2-Cu1-N1 = 81.30(11), N4-Cu1-N1 $=125.99(11)$, N2-Cu1-N3 $=122.66(11), N 4-C u 1-N 3=81.21(11)$, N1-Cu1-N3 $=127.62(11)^{\circ}$.

wrap around the metal centre (Fig. 6c). The isobutyl group containing $\mathrm{C} 1$ and $\mathrm{C} 2$ is disordered and has been modelled over two positions with site occupancies of 0.59 and 0.41 ; the carbon atom of the $\mathrm{CH}_{2}$ group is common to both positions. The distortion of the ligand containing $\mathrm{N} 3$ and N4 extends to the bromophenyl unit with Br4; the phenylpyridine unit is significantly bowed. The origin of the distortion is not clear but may be associated with interactions with the ordered $\left[\mathrm{PF}_{6}\right]^{-}$ ion which is sandwiched between the phenyl rings containing C52 and C58 with three short $\mathrm{CH} \cdots \mathrm{F}$ contacts in the range 2.59 and $2.71 \AA$. In addition, atom $\mathrm{Br} 4$ exhibits a short contact with $\mathrm{Br}^{\mathrm{i}}\left(\mathrm{Br} 4 \cdots \mathrm{Br} 2^{\mathrm{i}}=3.6922(7) \AA\right.$, symmetry code $\mathrm{i}=1-x, \frac{1}{2}+y$, $\frac{1}{2}-z$, van der Waals radius of $\left.\mathrm{Br}=1.95 \AA\right) .{ }^{36}$ As in the two previous structures, the extent that each phenylpyridine unit deviates from planarity is controlled by packing forces with twist angles lying in the range 20.1-40.3 ${ }^{\circ}$. The $\mathrm{Et}_{2} \mathrm{O}$ molecule is disordered and has been modelled over two sites on a special position.

Crystals of $\left[\mathrm{Cu}(5)_{2}\right]\left[\mathrm{PF}_{6}\right] \cdot \mathrm{CH}_{2} \mathrm{Cl}_{2}$ suitable for X-ray diffraction were grown by diffusion of $\mathrm{Et}_{2} \mathrm{O}$ into a $\mathrm{CH}_{2} \mathrm{Cl}_{2}$ solution of the complex. Fig. 8 shows the structure of the $\left[\mathrm{Cu}(5)_{2}\right]^{+}$cation and bond lengths and angles involving atom $\mathrm{Cu} 1$ are given in the caption. The complex crystallizes in the orthorhombic space group $\mathrm{Pbcn}$ with atom Cu1 lying on a two-fold axis. In contrast to $\left[\mathrm{Cu}(1)_{2}\right]^{+},\left[\mathrm{Cu}(2)_{2}\right]^{+}$and $\left[\mathrm{Cu}(3)_{2}\right]^{+}$, the $\left[\mathrm{Cu}(5)_{2}\right]^{+}$cation possesses a flattened structure (Fig. 5) with an angle between the least squares planes containing Cu1 and each bpy unit of only $44.1^{\circ}$. This is a consequence of a $\pi$-stacking interaction between each 6- (or 6'-) phenyl-substituent and a pyridine ring of the adjacent ligand. To achieve four such interactions, the bpy domains deviate from planarity (19.6 and $30.3^{\circ}$, respectively) and each phenyl ring twists ( 31.2 and $62.4^{\circ}$ ) with respect to the pyridine ring to which it is bonded. Despite these conformational changes, optimal $\pi$-stacking interactions with parallel rings are not achieved. The structural features of the copper(I) coordination sphere in $\left[\mathrm{Cu}(5)_{2}\right]^{+}$are consistent with the flattening observed in a range of $\left\{\mathrm{Cu}\left(6,6^{\prime}-\mathrm{Ph}_{2} \mathrm{bpy}\right)_{2}\right\}^{+}$,

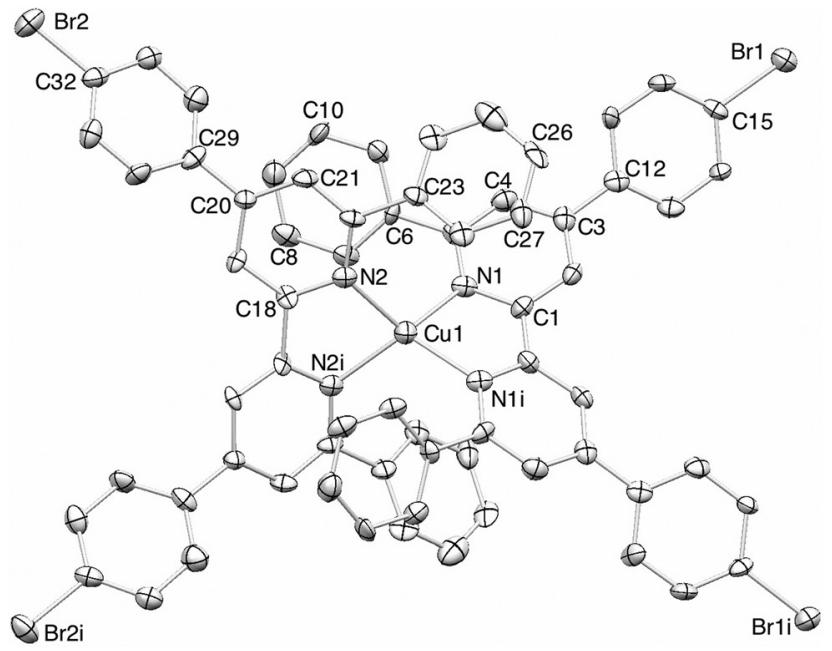

Fig. 8 ORTEP representation of the $\left[\mathrm{Cu}(\mathbf{5})_{2}\right]^{+}$cation in $\left[\mathrm{Cu}(\mathbf{5})_{2}\right]\left[\mathrm{PF}_{6}\right] \cdot \mathrm{CH}_{2} \mathrm{Cl}_{2}$ ( $\mathrm{H}$ atoms omitted and ellipsoids plotted at $30 \%$ probability level). Bond parameters in the coordination sphere: Cu1-N1 = 2.013(6), Cu1-N2 = 2.017(6) $\AA$; N1-Cu1$\mathrm{N}^{\mathrm{i}}=83.6(4), \mathrm{N} 1-\mathrm{Cu} 1-\mathrm{N} 2{ }^{\mathrm{i}}=138.5(2), \mathrm{N} 1-\mathrm{Cu} 1-\mathrm{N} 2=112.1(2), \mathrm{N} 2{ }^{\mathrm{i}}-\mathrm{Cu} 1-\mathrm{N} 2=$ $82.0(4)^{\circ}$. Symmetry code $\mathrm{i}=-x, y, 3 / 2-z$.

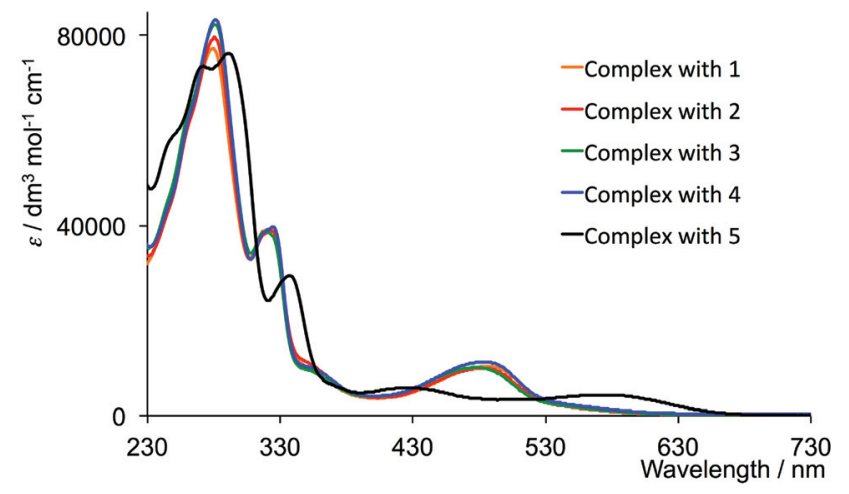

Fig. 9 Absorption spectra of $\mathrm{CH}_{2} \mathrm{Cl}_{2}$ solutions $\left(1.0 \times 10^{-5} \mathrm{~mol} \mathrm{dm}{ }^{-3}\right)$ of $\left[\mathrm{CuL}_{2}\right]-$ $\left[\mathrm{PF}_{6}\right]$ for $\mathrm{L}=\mathbf{1 - 5}$.

$\left\{\mathrm{Cu}\left(2,9-\mathrm{Ph}_{2} \text { phen }\right)_{2}\right\}$ and related complexes, ${ }^{20,21,37-50}$ although within these examples, the efficiency of the intra-cation $\pi$-interactions is variable. In $\left[\mathrm{Cu}(5)_{2}\right]\left[\mathrm{PF}_{6}\right] \cdot \mathrm{CH}_{2} \mathrm{Cl}_{2}$, face-to-face $\pi$-stacking occurs between pendant phenyl rings of neighbouring cations leading to columns of $\left[\mathrm{Cu}(5)_{2}\right]^{+}$cations running parallel to the $a$-axis. Additional packing interactions involve short $\mathrm{Br} \cdots \mathrm{Br}$ contacts, and close $\mathrm{CH} \cdots \mathrm{F}$ and $\mathrm{CH} \cdots \mathrm{Cl}$ contacts; both the $\left[\mathrm{PF}_{6}\right]^{-}$ion and the $\mathrm{CH}_{2} \mathrm{Cl}_{2}$ molecule are ordered.

The solution electronic absorption spectra of $\left[\mathrm{CuL}_{2}\right]\left[\mathrm{PF}_{6}\right]$ with $\mathrm{L}=1-5$ are shown in Fig. 9. Spectra for the complexes containing the aliphatic side chains are virtually superimposable with high energy bands assigned to ligand-based $\pi^{*} \leftarrow \pi$ transitions and a broad MLCT band in the visible region $\left(\lambda_{\max }\right.$ $=481-488 \mathrm{~nm}$ ). The dual bands observed in the visible region of the spectrum $\left[\mathrm{Cu}(5)_{2}\right]\left[\mathrm{PF}_{6}\right]$ and the red-shift to $573 \mathrm{~nm}$ are consistent with features in the absorption spectrum of $\left[\mathrm{Cu}(\mathrm{dpp})_{2}\right]^{+}(\mathrm{dpp}=$ 2,9-diphenyl-1,10-phenanthroline $)$ which 
are explained in terms of the flattened, low-symmetry structure of the complex. ${ }^{9,20}$ The spectrum of $\left[\mathrm{Cu}(5)_{2}\right]\left[\mathrm{PF}_{6}\right]$ retains the enhanced spectral response in the higher energy bands observed for ligand 5 compared to 1-4 (Fig. 1).

All the complexes are electrochemically active and cyclic voltammetric data are presented in Table 1. It is well established that in copper(I) diimine complexes, the presence of substituents adjacent to the N,N-donor sites (e.g. 6,6'-positions in 2,2'bpy or 2,9-sites in 1,10-phenanthroline) stabilizes copper(I) with respect to oxidation by sterically hindering the flattening of the coordination sphere that accompanies the $\mathrm{d}^{10}\left(\mathrm{Cu}^{+}\right)$to $\mathrm{d}^{9}\left(\mathrm{Cu}^{2+}\right)$ electronic configurational change. ${ }^{19,22}$ Cyclic voltammograms were recorded in $\mathrm{CH}_{2} \mathrm{Cl}_{2}$ to avoid problems associated with coordinating solvents such as MeCN. ${ }^{19}$ The potentials for the metal-centred oxidation process in $\left[\mathrm{CuL}_{2}\right]$ $\left[\mathrm{PF}_{6}\right]$ with $\mathrm{L}=\mathbf{1 - 4}$ follow the expected trend, with the complex containing the least sterically demanding (methyl) substituents being the easiest to oxidize: $\left[\mathrm{Cu}(\mathbf{1})_{2}\right]^{+}<\left[\mathrm{Cu}(2)_{2}\right]^{+} \approx$ $\left[\mathrm{Cu}(3)_{2}\right]^{+} \approx\left[\mathrm{Cu}(\mathbf{4})_{2}\right]^{+}$. The oxidation potential for $\left[\mathrm{Cu}(\mathbf{1})_{2}\right]\left[\mathrm{PF}_{6}\right]$ $(+0.42 \mathrm{~V})$ compares to $+0.50 \mathrm{~V}\left(v s . \mathrm{Fc} / \mathrm{Fc}^{+}\right.$in $\left.\mathrm{CH}_{2} \mathrm{Cl}_{2}\right)$ for $\left[\mathrm{Cu}(\mathrm{dmp})_{2}\right]\left[\mathrm{PF}_{6}\right]$ (dmp = 2,9-dimethyl-1,10-phenanthroline).

Table 1 Cyclic voltammetric data for $\left[\mathrm{CuL}_{2}\right]\left[\mathrm{PF}_{6}\right]$ with $\mathrm{L}=\mathbf{1 - 5}$ with respect to $\mathrm{Fc} / \mathrm{Fc}^{+} ; \mathrm{CH}_{2} \mathrm{Cl}_{2}$ solutions with $\left[{ }^{n} \mathrm{Bu}_{4} \mathrm{~N}\right]\left[\mathrm{PF}_{6}\right]$ as the supporting electrolyte and a scan rate of $0.1 \mathrm{~V} \mathrm{~s}^{-1}$. Processes are reversible unless otherwise stated (ir = irreversible)

\begin{tabular}{llll}
\hline Complex & $E_{1 / 2}^{\mathrm{ox}} / \mathrm{V}\left(E_{\mathrm{pc}}-E_{\mathrm{pa}} / \mathrm{mV}\right)$ & $E_{1 / 2}^{\mathrm{ox}} / \mathrm{V}$ & $E_{1 / 2}^{\mathrm{red}} / \mathrm{V}$ \\
\hline$\left[\mathrm{Cu}(1)_{2}\right]\left[\mathrm{PF}_{6}\right]$ & $+0.42(94)$ & $+1.49^{\mathrm{ir}}$ & $-2.16^{\mathrm{ir}}$ \\
{$\left[\mathrm{Cu}(2)_{2}\right]\left[\mathrm{PF}_{6}\right]$} & $+0.52(87)$ & $+1.47^{\mathrm{ir}}$ & $-2.16^{\mathrm{ir}}$ \\
{$\left[\mathrm{Cu}(3)_{2}\right]\left[\mathrm{PF}_{6}\right]$} & $+0.54(96)$ & $+1.47^{\mathrm{ir}}$ & $-2.20^{\mathrm{ir}}$ \\
{$\left[\mathrm{Cu}(4)_{2}\right]\left[\mathrm{PF}_{6}\right]$} & $+0.54(84)$ & $+1.49^{\mathrm{ir}}$ & $-2.14^{\mathrm{ir}}$ \\
{$\left[\mathrm{Cu}(5)_{2}\right]\left[\mathrm{PF}_{6}\right]$} & $+0.36(86)$ & - & $a$
\end{tabular}

${ }^{a}$ No reduction process observed within the solvent accessible window.

Table 2 Sealed and masked DSC cell performances using anchoring ligands $\mathbf{6}$ and $\mathbf{7}$ with respect to standard dye N719 measured under the same conditions

On the day of sealing the cell

\begin{tabular}{|c|c|c|c|c|c|c|}
\hline \multirow[b]{2}{*}[\mathrm{CuL}_{2}]{$^{+}$} & \multirow[b]{2}{*}{ Anchor } & \\
\hline & & $J_{\mathrm{SC}} / \mathrm{mA} \mathrm{cm}^{-2}$ & $V_{\mathrm{OC}} / \mathrm{mV}$ & ff & $\eta / \%$ & Relative $\eta / \%$ \\
\hline$\left[\mathrm{Cu}(\mathbf{1})_{2}\right]^{+}$ & 6 & 3.21 & 477 & 0.72 & 1.10 & 16.6 \\
\hline$\left[\mathrm{Cu}(2)_{2}\right]^{+}$ & 6 & 3.64 & 478 & 0.71 & 1.24 & 18.8 \\
\hline$\left[\mathrm{Cu}(3)_{2}\right]^{+}$ & 6 & 4.32 & 515 & 0.69 & 1.54 & 23.2 \\
\hline$\left[\mathrm{Cu}(\mathbf{4})_{2}\right]^{+}$ & 6 & 3.57 & 475 & 0.71 & 1.21 & 18.3 \\
\hline$\left[\mathrm{Cu}(\mathbf{5})_{2}\right]^{+}$ & 6 & 0.35 & 473 & 0.56 & 0.09 & 1.38 \\
\hline$\left[\mathrm{Cu}(\mathbf{1})_{2}\right]^{+}$ & 7 & 5.09 & 497 & 0.72 & 1.82 & 27.5 \\
\hline$\left[\mathrm{Cu}(2)_{2}\right]^{+}$ & 7 & 5.50 & 497 & 0.71 & 1.95 & 29.5 \\
\hline$\left[\mathrm{Cu}(3)_{2}\right]^{+}$ & 7 & 6.73 & 541 & 0.70 & 2.55 & 38.5 \\
\hline$\left[\mathrm{Cu}(4)_{2}\right]^{+}$ & 7 & 5.07 & 507 & 0.71 & 1.83 & 27.7 \\
\hline$\left[\mathrm{Cu}(\mathbf{5})_{2}\right]^{+}$ & 7 & 2.74 & 605 & 0.66 & 1.10 & 16.6 \\
\hline N719 & & 15.06 & 634 & 0.69 & 6.62 & 100.0 \\
\hline
\end{tabular}

3 days after sealing the cell

\begin{tabular}{|c|c|c|c|c|c|c|}
\hline \multirow[b]{2}{*}[\mathrm{CuL}_{2}]{$^{+}$} & \multirow[b]{2}{*}{ Anchor } & \\
\hline & & $J_{\mathrm{SC}} / \mathrm{mA} \mathrm{cm}^{-2}$ & $V_{\mathrm{OC}} / \mathrm{mV}$ & ff & $\eta / \%$ & Relative $\eta / \%$ \\
\hline$\left[\mathrm{Cu}(\mathbf{1})_{2}\right]^{+}$ & 6 & 4.44 & 500 & 0.72 & 1.60 & 26.3 \\
\hline$\left[\mathrm{Cu}(2)_{2}\right]^{+}$ & 6 & 3.51 & 506 & 0.67 & 1.19 & 19.5 \\
\hline$\left[\mathrm{Cu}(3)_{2}\right]^{+}$ & 6 & 4.35 & 543 & 0.65 & 1.54 & 25.3 \\
\hline$\left[\mathrm{Cu}(\mathbf{4})_{2}\right]^{+}$ & 6 & 3.97 & 505 & 0.70 & 1.40 & 23.0 \\
\hline$\left[\mathrm{Cu}(\mathbf{5})_{2}\right]^{+}$ & 6 & 2.22 & 534 & 0.68 & 0.81 & 13.3 \\
\hline$\left[\mathrm{Cu}(\mathbf{1})_{2}\right]^{+}$ & 7 & 5.85 & 519 & 0.72 & 2.19 & 36.0 \\
\hline$\left[\mathrm{Cu}(2)_{2}\right]^{+}$ & 7 & 5.17 & 524 & 0.67 & 1.82 & 29.9 \\
\hline$\left[\mathrm{Cu}(3)_{2}\right]^{+}$ & 7 & 7.02 & 566 & 0.63 & 2.49 & 40.8 \\
\hline$\left[\mathrm{Cu}(4)_{2}\right]^{+}$ & 7 & 5.54 & 496 & 0.68 & 1.87 & 30.6 \\
\hline$\left[\mathrm{Cu}(\mathbf{5})_{2}\right]^{+}$ & 7 & 6.41 & 600 & 0.73 & 2.80 & 45.9 \\
\hline N719 & & 14.80 & 673 & 0.61 & 6.09 & 100.0 \\
\hline
\end{tabular}

4 days after sealing the cell

\begin{tabular}{|c|c|c|c|c|c|c|}
\hline \multirow[b]{2}{*}[\mathrm{CuL}_{2}]{$^{+}$} & \multirow[b]{2}{*}{ Anchor } & \\
\hline & & $J_{\mathrm{SC}} / \mathrm{mA} \mathrm{cm}^{-2}$ & $V_{\mathrm{OC}} / \mathrm{mV}$ & $\mathrm{ff}$ & $\eta / \%$ & Relative $\eta / \%$ \\
\hline$\left[\mathrm{Cu}(\mathbf{1})_{2}\right]^{+}$ & 6 & 4.53 & 507 & 0.72 & 1.65 & 27.7 \\
\hline$\left[\mathrm{Cu}(2)_{2}\right]^{+}$ & 6 & 3.56 & 502 & 0.66 & 1.18 & 19.8 \\
\hline$\left[\mathrm{Cu}(3)_{2}\right]^{+}$ & 6 & 4.44 & 549 & 0.66 & 1.61 & 27.0 \\
\hline$\left[\mathrm{Cu}(4)_{2}\right]^{+}$ & 6 & 4.21 & 514 & 0.69 & 1.50 & 25.2 \\
\hline$\left[\mathrm{Cu}(\mathbf{5})_{2}\right]^{+}$ & 6 & 3.53 & 536 & 0.68 & 1.29 & 21.6 \\
\hline$\left[\mathrm{Cu}(\mathbf{1})_{2}\right]^{+}$ & 7 & 6.01 & 527 & 0.73 & 2.30 & 38.6 \\
\hline$\left[\mathrm{Cu}(2)_{2}\right]^{+}$ & 7 & 5.30 & 525 & 0.65 & 1.80 & 30.2 \\
\hline$\left[\mathrm{Cu}(3)_{2}\right]^{+}$ & 7 & 7.06 & 571 & 0.60 & 2.43 & 40.8 \\
\hline$\left[\mathrm{Cu}(4)_{2}\right]^{+}$ & 7 & 6.07 & 520 & 0.60 & 1.90 & 31.9 \\
\hline$\left[\mathrm{Cu}(5)_{2}\right]^{+}$ & 7 & 6.70 & 592 & 0.73 & 2.89 & 48.5 \\
\hline N719 & & 14.66 & 679 & 0.60 & 5.96 & 100.0 \\
\hline
\end{tabular}


Table 3 DSC performance data for an independent set of sealed and masked cells using anchoring ligand 7; data are with respect to standard dye N719 measured under the same conditions

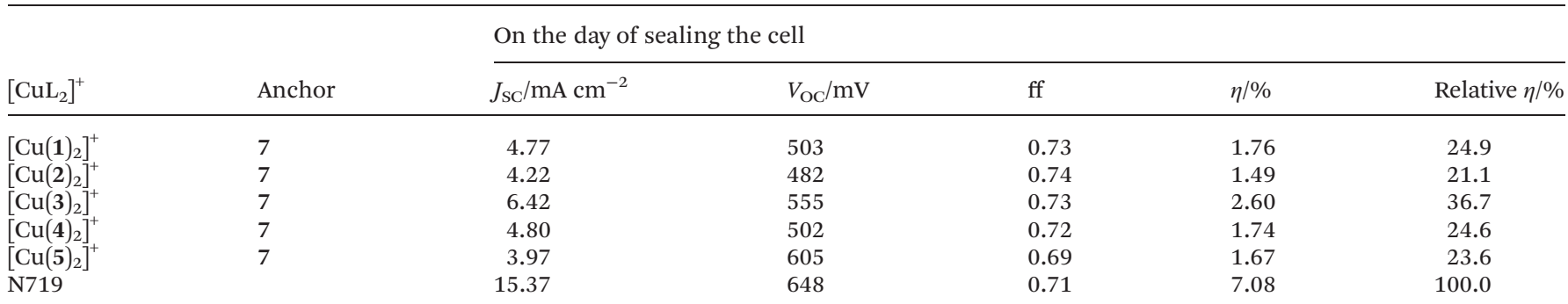

1 day after sealing the cell

\begin{tabular}{|c|c|c|c|c|c|c|}
\hline$\left[\mathrm{CuL}_{2}\right]^{+}$ & Anchor & $J_{\mathrm{SC}} / \mathrm{mA} \mathrm{cm}^{-2}$ & $V_{\mathrm{OC}} / \mathrm{mV}$ & $\mathrm{ff}$ & $\eta / \%$ & Relative $\eta / \%$ \\
\hline$\left[\mathrm{Cu}(\mathbf{1})_{2}\right]^{+}$ & 7 & 6.09 & 520 & 0.74 & 2.33 & 28.4 \\
\hline$\left[\mathrm{Cu}(2)_{2}\right]^{+}$ & 7 & 5.61 & 499 & 0.74 & 2.06 & 25.1 \\
\hline$\left[\mathrm{Cu}(3)_{2}\right]^{+}$ & 7 & 6.74 & 571 & 0.73 & 2.81 & 34.2 \\
\hline$\left[\mathrm{Cu}(\mathbf{4})_{2}\right]^{+}$ & 7 & 5.92 & 510 & 0.73 & 2.19 & 26.6 \\
\hline$\left[\mathrm{Cu}(5)_{2}\right]^{+}$ & 7 & 6.81 & 611 & 0.72 & 3.01 & 36.6 \\
\hline N719 & & 17.10 & 677 & 0.71 & 8.22 & 100.0 \\
\hline
\end{tabular}

2 days after sealing the cell

\begin{tabular}{|c|c|c|c|c|c|c|}
\hline$\left[\mathrm{CuL}_{2}\right]^{+}$ & Anchor & $J_{\mathrm{SC}} / \mathrm{mA} \mathrm{cm}^{-2}$ & $V_{\mathrm{OC}} / \mathrm{mV}$ & $\mathrm{ff}$ & $\eta / \%$ & Relative $\eta / \%$ \\
\hline$\left[\mathrm{Cu}(\mathbf{1})_{2}\right]^{+}$ & 7 & 5.93 & 525 & 0.74 & 2.31 & 27.8 \\
\hline$\left[\mathrm{Cu}(2){ }_{2}\right]^{+}$ & 7 & 5.42 & 501 & 0.73 & 1.99 & 24.0 \\
\hline$\left[\mathrm{Cu}(3)_{2}\right]^{+}$ & 7 & 6.91 & 576 & 0.72 & 2.87 & 34.6 \\
\hline$\left[\mathrm{Cu}(\mathbf{4})_{2}\right]^{+}$ & 7 & 5.51 & 521 & 0.73 & 2.09 & 25.2 \\
\hline$\left[\mathrm{Cu}(5)_{2}\right]^{+}$ & 7 & 6.96 & 606 & 0.74 & 3.10 & 37.4 \\
\hline N719 & & 17.2 & 684 & 0.71 & 8.30 & 100.0 \\
\hline
\end{tabular}

7 days after sealing the cell

\begin{tabular}{|c|c|c|c|c|c|c|}
\hline \multirow[b]{2}{*}[\mathrm{CuL}_{2}]{$^{+}$} & \multirow[b]{2}{*}{ Anchor } & \\
\hline & & $J_{\mathrm{SC}} / \mathrm{mA} \mathrm{cm}^{-2}$ & $V_{\mathrm{OC}} / \mathrm{mV}$ & ff & $\eta / \%$ & Relative $\eta / \%$ \\
\hline$\left[\mathrm{Cu}(\mathbf{1})_{2}\right]^{+}$ & 7 & 5.73 & 530 & 0.74 & 2.26 & 26.8 \\
\hline$\left[\mathrm{Cu}(2)_{2}\right]^{+}$ & 7 & 5.51 & 513 & 0.74 & 2.09 & 24.7 \\
\hline$\left[\mathrm{Cu}(\mathbf{3})_{2}\right]^{+}$ & 7 & 6.69 & 584 & 0.73 & 2.85 & 33.7 \\
\hline$\left[\mathrm{Cu}(4)_{2}\right]^{+}$ & 7 & 5.44 & 531 & 0.71 & 2.05 & 24.3 \\
\hline$\left[\mathrm{Cu}(\mathbf{5})_{2}\right]^{+}$ & 7 & 7.03 & 590 & 0.73 & 3.01 & 35.6 \\
\hline N719 & & 17.23 & 696 & 0.71 & 8.45 & 100.0 \\
\hline
\end{tabular}

Oxidation of the copper(I) ion in $\left[\mathrm{Cu}(5)_{2}\right]^{+}$occurs at lower potential than in $\left[\mathrm{Cu}(\mathbf{1})_{2}\right]^{+}$, and the value of $+0.36 \mathrm{~V}$ is similar to that reported for $\left[\mathrm{CuL}_{2}\right]^{+}$where $\mathrm{L}=4,4^{\prime}, 6,6^{\prime}$-tetraphenyl-2,2'bipyridine $\left(+0.39 \mathrm{~V}\right.$ vs. $\mathrm{Fc} / \mathrm{Fc}^{+}$in $\mathrm{CH}_{2} \mathrm{Cl}_{2}$, corrected ${ }^{51}$ from the published value $^{40}$ of $+0.77 \mathrm{~V} v s$. SCE). The structural reorganization that accompanies metal oxidation is less pronounced in $\left[\mathrm{Cu}(\mathbf{5})_{2}\right]^{+}$than in $\left[\mathrm{Cu}(\mathbf{1})_{2}\right]^{+}$. This assumption is based upon the flattened geometry in the former brought about by $\pi$-stacking interactions that occur between the phenyl groups of one ligand and the bpy domain of the other. This has been observed in a number of related structures. ${ }^{37-41}$ Each of the complexes containing aliphatic substituents exhibits an irreversible oxidation process at higher potential (Table 1) most likely associated with the oxidation of the 4-bromophenyl substituent to the corresponding radical cation. ${ }^{52}$

\section{Performance of the dyes in DSCs}

Heteroleptic dyes were assembled by stepwise adsorption of the anchoring ligand 6 or 7 onto $\mathrm{TiO}_{2}$ followed by treatment with the labile ${ }^{53,54}$ homoleptic complexes $\left[\mathrm{CuL}_{2}\right]\left[\mathrm{PF}_{6}\right](\mathrm{L}=\mathbf{1 - 5})$ resulting in ligand exchange and surface immobilisation of the heteroleptic dyes $[\mathrm{Cu}(\mathrm{L})(6)]^{+}$and $[\mathrm{Cu}(\mathrm{L})(7)]^{+}$shown in Scheme 2 . The proximity to the surface of the copper(I) centre and of the 6,6'-substituents in the ancillary ligand is related to the presence or not of the aryl spacer in the anchoring ligand. All cells were fully masked to prevent overestimation of conversion efficiency. ${ }^{55}$ Table 2 summarizes the DSC characteristics measured for each cell over a period of 4 days and with respect to the standard dye $\mathrm{N} 719$ (in the table, $V_{\text {OC }}=$ open circuit voltage, $J_{\mathrm{SC}}=$ short circuit current density, ff = fill factor). The final column of the table shows the energy conversion efficiencies relative to N719 which is arbitrarily set to $100 \%$.

The surface-bound heteroleptic dyes $[\mathrm{Cu}(\mathbf{1})(\mathbf{6})]^{+},[\mathrm{Cu}(\mathbf{2})(\mathbf{6})]^{+}$, $[\mathrm{Cu}(3)(6)]^{+}$and $[\mathrm{Cu}(4)(6)]^{+}$show a gain in values of $J_{\mathrm{SC}}$ and $V_{\mathrm{OC}}$ over the 4 day period, but overall there is little change to the fill factor for each cell. Of the four alkyl-substituted ligands, 3 (with isobutyl substituents) produces the most efficient dye with a relative energy efficiency, $\eta$, reaching $25.2 \%$ with respect 


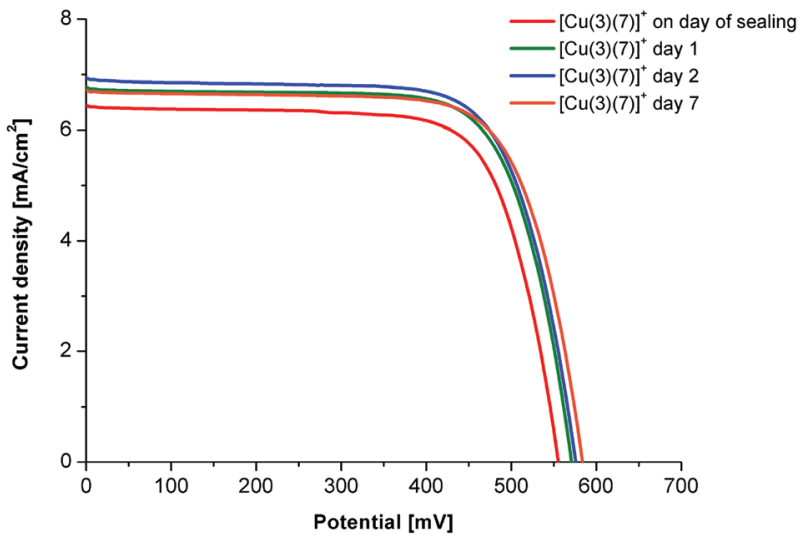

(a)

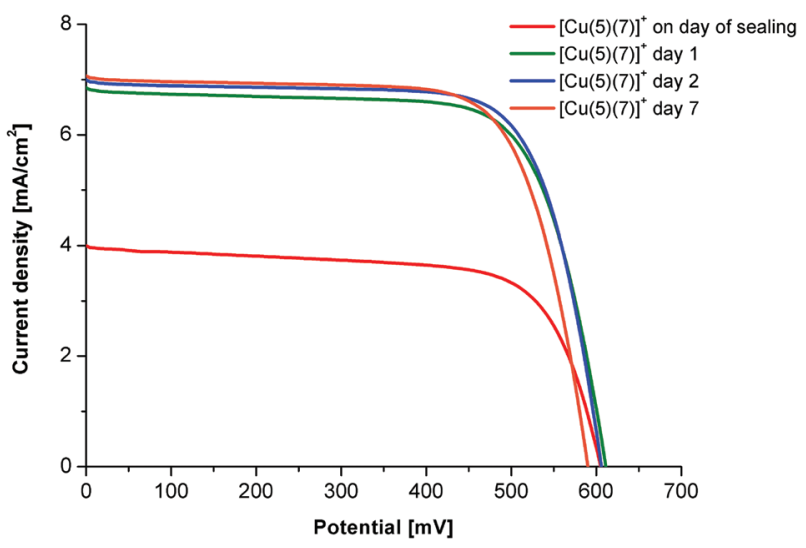

(b)

Fig. 10 Current density $\left(J_{S C}\right)$ against open-circuit voltage $\left(V_{\mathrm{OC}}\right)$ curves for the dyes (a) $[\mathrm{Cu}(\mathbf{3})(\mathbf{7})]^{+}$and (b) $[\mathrm{Cu}(\mathbf{5})(\mathbf{7})]^{+}$over a period of 7 days.

to N719 measured under the same conditions. The efficiency of the dye is significantly improved by incorporating the aryl spacer into the anchoring ligand (i.e. going from $[\mathrm{Cu}(3)(6)]^{+}$to $[\mathrm{Cu}(3)(7)]^{+}$), and after 4 days, the cell performs with a relative $\eta$ of $40.8 \%$ (Table 2). A comparison of the values of $J_{\mathrm{SC}}, V_{\mathrm{OC}}$ and $\eta$ in Table 2 for a given dye containing 1, 2, 3 or 4 over the 7 day period reveals a small enhancement as the cell initially matures. Unexpectedly, the DSCs containing ligand 5 performed poorly on the first day, but as Table 2 shows, energy conversion efficiency improved considerably as the cell aged. This is mainly due to an increase in the short-circuit current density, for both $[\mathrm{Cu}(5)(6)]^{+}$and $[\mathrm{Cu}(5)(7)]^{+}$. Once again, the presence of the aryl spacer in the phosphonic acid anchoring ligand is beneficial and a relative efficiency of $48.4 \%$ was achieved, compared to $21.6 \%$ without the spacer. The highly promising performances of dyes with the diphenyl-substituted ancillary ligand $\mathbf{5}$ are of interest in the context of recent findings of Chen and coworkers. ${ }^{8}$ Their key conclusions are that the photoinduced structural changes that occur to $\left[\mathrm{Cu}(\mathrm{dpp})_{2}\right]^{+}$ ( $\mathrm{dpp}=2,9$-diphenyl-1,10-phenanthroline) are small, and that changes in the orientations of the phenyl rings upon excitation result in a copper centre protected from solvent attack, thereby prolonging the MLCT lifetime in coordinating solvents. Chen and coworkers also conclude that electronic charge transferred from copper to dpp ligands does not reside on the phenyl rings since conjugation between phen and $\mathrm{Ph}$ domains is lost when the ligand changes its conformation. In our case, there is an additional degree of freedom associated with the rotation about the interannular $\mathrm{C}-\mathrm{C}$ bond of the by ligand.

In order to check the reproducibility of our results, a new set of masked solar cells was prepared using anchoring ligand 7, and cell performances were measured over a 7 day period (Table 3). A comparison of the data in Tables 2 and 3 reveals analogous trends, although absolute efficiencies are lower for the second batch of cells than for the first. Fig. 10a shows plots of current density against open-circuit voltage for $[\mathrm{Cu}(3)(7)]^{+}$, illustrating the initial maturing of the cell, followed by consistent behaviour for a week. The ripening process of the cell containing $[\mathrm{Cu}(5)(7)]^{+}$is illustrated in the $I-V$ curve in Fig. $10 \mathrm{~b}$ and replicates that observed for the first set of cells. Good fill factors were obtained for all cells in both sets (Tables 2 and 3).

The incident photon-to-current conversion efficiency (IPCE) or external quantum efficiency (EQE) spectra were measured for each of the solar cells detailed in Table 3. The spectra shown in Fig. 11a for $[\mathrm{Cu}(3)(7)]^{+}$are representative of those of cells containing the dyes $[\mathrm{Cu}(\mathbf{1})(7)]^{+},[\mathrm{Cu}(2)(7)]^{+},[\mathrm{Cu}(\mathbf{3})(7)]^{+}$and $[\mathrm{Cu}(4)(7)]^{+}$. The peak maxima in the IPCE spectra at $\approx 470 \mathrm{~nm}$ correlate with the observed maxima $\left(\lambda_{\max }=481-488 \mathrm{~nm}\right)$ in the solution absorption spectra of the homoleptic complexes $\left[\mathrm{Cu}(\mathbf{1})_{2}\right]^{+},\left[\mathrm{Cu}(2)_{2}\right]^{+},\left[\mathrm{Cu}(3)_{2}\right]^{+}$and $\left[\mathrm{Cu}(4)_{2}\right]^{+}$(Fig. 9). IPCE peak maxima of between 39 and $42 \%$ are achieved for $[\mathrm{Cu}(1)(7)]^{+}$, $38-40 \%$ for $[\mathrm{Cu}(2)(7)]^{+}, 47-50 \%$ for $[\mathrm{Cu}(3)(7)]^{+}$and $38-39 \%$ for $[\mathrm{Cu}(4)(7)]^{+}$, corresponding to the trends in efficiencies given in Table 3. Fig. 11b shows the IPCE spectra for $[\mathrm{Cu}(5)(7)]^{+}$from the day of sealing to a week after sealing the cell. Initially an IPCE maximum of $28 \%$ is observed but, consistent with the ripening of the cell described above, this increases to $46 \%$ after a week. It is noticeable that the shape of the IPCE curve for $[\mathrm{Cu}(5)(7)]^{+}$is close to those of the other complexes, i.e. the differences observed in the solution absorption spectra between the homoleptic complexes containing alkyl and phenyl substituents are not replicated in the IPCE curves of the heteroleptic complexes. To gain more insight into this, the absorption spectra of the surface-anchored heteroleptic dyes $[\mathrm{Cu}(3)(7)]^{+}$and $[\mathrm{Cu}(5)(7)]^{+}$were recorded. The anodes were prepared as described in the Experimental section (solar cell fabrication), and their absorption spectra are shown in Fig. 12. The absorption spectrum of the photoanode with adsorbed $[\mathrm{Cu}(5)(7)]^{+}$also exhibits an enhanced spectral response at $c a$. $600 \mathrm{~nm}$ as also seen in the homoleptic complex $\left[\mathrm{Cu}(5)_{2}\right]^{+}$in solution (Fig. 9).

Fig. 13 compares the IPCE spectra of cells containing the five copper(I)-containing dyes with that of N719. The IPCE curves indicate improved response in the red region of the spectrum consistent with the red-shifting of the absorption spectra of the dyes containing ancillary ligand 5. While good photon-to-current quantum efficiency is achieved with the copper(I) dyes over the range $450-550 \mathrm{~nm}$, the dyes do not 


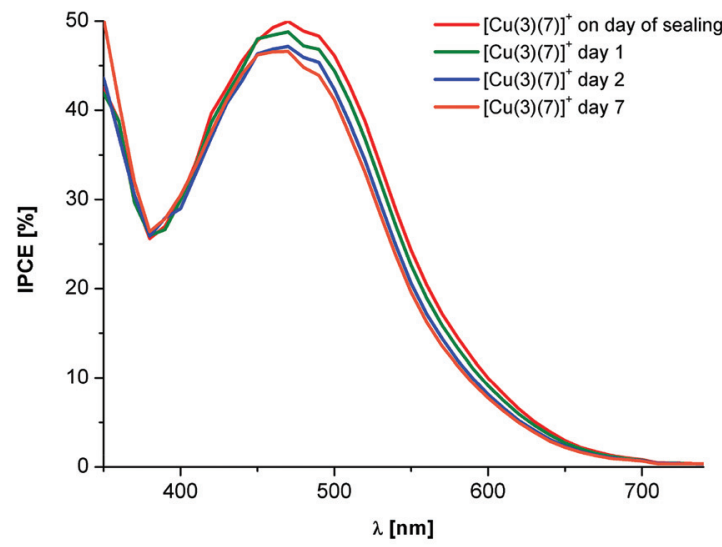

(a)

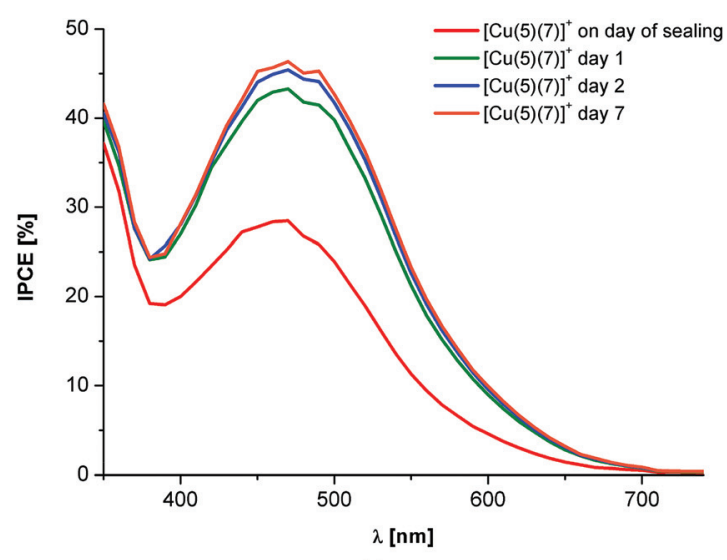

(b)

Fig. 11 IPCE spectra for DSCs containing the anchored dyes (a) $[\mathrm{Cu}(\mathbf{3})(\mathbf{7})]^{+}$and (b) $[\mathrm{Cu}(\mathbf{5})(\mathbf{7})]^{+}$over a period of a week after sealing the solar cells.

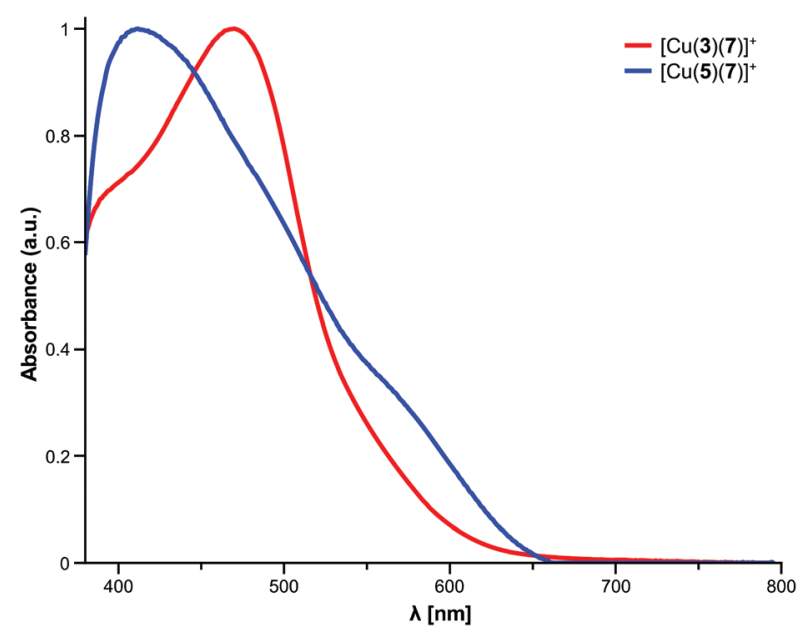

Fig. 12 Normalized absorption spectra of photoanodes with surface-anchored heteroleptic dyes $[\mathrm{Cu}(\mathbf{3})(\mathbf{7})]\left[\mathrm{PF}_{6}\right]$ and $[\mathrm{Cu}(\mathbf{5})(\mathbf{7})]\left[\mathrm{PF}_{6}\right]$.

achieve the broad spectra response exhibited by the state-ofthe-art ruthenium(II) dye N719. Specifically, aspects of molecular design that need to be addressed include the further red-

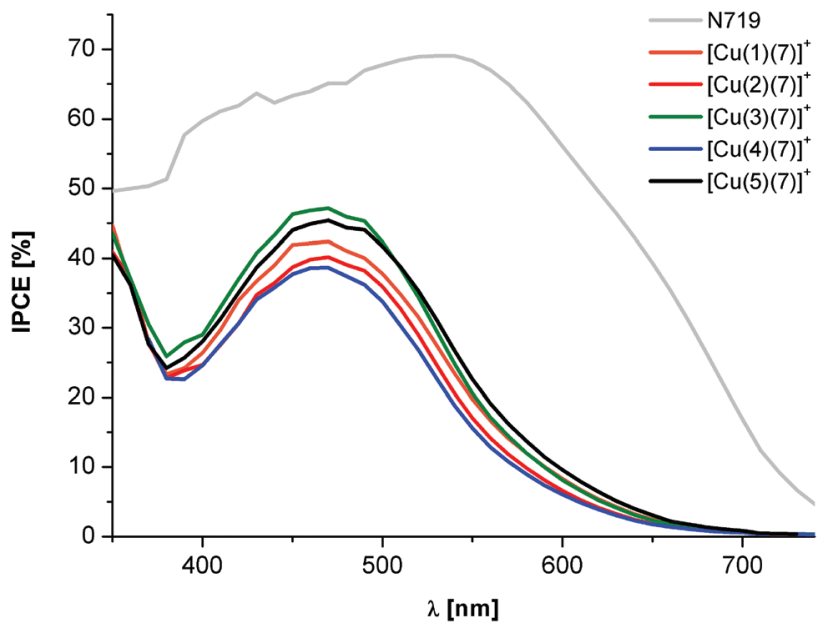

Fig. 13 IPCE spectra of cells containing the copper(I)-containing dyes compared to a cell with standard dye N719.

shifting of the absorption and also an increase in the absorptivity.

\section{Conclusions}

We have described the syntheses and characterizations of the homoleptic complexes $\left[\mathrm{CuL}_{2}\right]\left[\mathrm{PF}_{6}\right]$ with $\mathrm{L}=\mathbf{1 - 5}$. The solid state structures of $2\left\{\left[\mathrm{Cu}(\mathbf{1})_{2}\right]\left[\mathrm{PF}_{6}\right]\right\} \cdot 3 \mathrm{Me}_{2} \mathrm{CO},\left[\mathrm{Cu}(2)_{2}\right]\left[\mathrm{PF}_{6}\right]$, and $2\left\{\left[\mathrm{Cu}(3)_{2}\right]\left[\mathrm{PF}_{6}\right]\right\} \cdot \mathrm{Et}_{2} \mathrm{O}$ confirm distorted tetrahedral environments for the $\mathrm{Cu}^{+}$ions. In $\left[\mathrm{Cu}(5)_{2}\right]\left[\mathrm{PF}_{6}\right] \cdot \mathrm{CH}_{2} \mathrm{Cl}_{2}$, the cation possesses a significantly flattened geometry caused by $\pi$-interactions between the pendant phenyl rings and bpy domains of adjacent ligands. The solution absorption spectrum of each of $\left[\mathrm{Cu}(\mathbf{1})_{2}\right]\left[\mathrm{PF}_{6}\right],\left[\mathrm{Cu}(2)_{2}\right]\left[\mathrm{PF}_{6}\right],\left[\mathrm{Cu}(3)_{2}\right]\left[\mathrm{PF}_{6}\right]$ and $\left[\mathrm{Cu}(\mathbf{4})_{2}\right]\left[\mathrm{PF}_{6}\right]$ shows an MLCT band at around $485 \mathrm{~nm}$. Greater absorptivity in the red region of the spectrum is achieved by replacing the 6- and 6'-alkyl substituents with phenyl groups in the complex $\left[\mathrm{Cu}(5)_{2}\right]\left[\mathrm{PF}_{6}\right]$ and results in the appearance of two broad bands in the visible region, the longer wavelength band at $573 \mathrm{~nm}$. The efficiencies, $I-V$ curves and IPCE spectra of DSCs containing photoanodes with $\left[\mathrm{Cu}(\mathrm{L})\left(\mathrm{L}_{\text {anchor }}\right)\right]^{+}$dyes in which $\mathrm{L}$ is $\mathbf{1 - 5}$ and $L_{\text {anchor }}$ is 6 or 7 have been measured. Cell performance is enhanced by (i) an aromatic spacer in the anchoring ligand (i.e. anchoring ligand 7 vs. 6), and (ii) either iso butyl or phenyl substituents in the 6- and 6'-positions of the ancillary ligand, L. The highest energy-conversion efficiencies are observed for dyes $[\mathrm{Cu}(3)(7)]^{+}(\eta=2.43 \%$ compared to $5.96 \%$ for standard dye N719) and $[\mathrm{Cu}(5)(7)]^{+}(\eta=2.89 \%$ compared to $5.96 \%$ for N719). It is important to note that cells incorporating $[\mathrm{Cu}(5)(7)]^{+}$undergo a ripening process leading to an optimum performance only after a number of days.

\section{Acknowledgements}

We thank the European Research Council (Advanced Grant 267816 LiLo), the Swiss National Science Foundation, the 
Swiss Nanoscience Institute and the University of Basel for financial support. Highfield NMR spectra were recorded by Nik Hostettler, Cathrin Ertl or Dr. Colin Martin; Ewald Schönhofer and Annika Büttner prepared and screenprinted the mesoporous $\mathrm{TiO}_{2}$. Liselotte Siegfried is thanked for assisting with UV-vis measurements of the surfaceanchored dyes.

\section{Notes and references}

1 B. Bozic-Weber, E. C. Constable and C. E. Housecroft, Coord. Chem. Rev., 2013, DOI: 10.1016/j.ccr.2013.05.019.

2 M. Sandroni, M. Kayanuma, A. Planchat, N. Szuwarski, E. Blart, Y. Pellegrin, C. Daniel, M. Boujtita and F. Odobel, Dalton Trans., 2013, DOI: 10.1039/C3DT50852H.

3 N. Alonso-Vante, J.-F. Nierengarten and J.-P. Sauvage, J. Chem. Soc., Dalton Trans., 1994, 1650.

4 N. Armaroli, Chem. Soc. Rev., 2001, 30, 113.

5 N. Armaroli, Top. Curr. Chem., 2007, 280, 69.

6 X. Lu, C.-M. L. Wu, S. Wei and W. Guo, J. Phys. Chem. A, 2010, 114, 1178.

7 X. Lu, S. Wei, C.-M. L. Wu, S. Li and W. Guo, J. Phys. Chem. C, 2011, 115, 3753.

8 M. W. Mara, N. E. Jackson, J. Huang, A. B. Stickrath, X. Zhang, N. A. Gothard, M. A. Ratner and L. X. Chen, J. Phys. Chem. B, 2013, 117, 1921.

9 B. Bozic-Weber, V. Chaurin, E. C. Constable, C. E. Housecroft, M. Meuwly, M. Neuburger, J. A. Rudd, E. Schönhofer and L. Siegfried, Dalton Trans., 2012, 41, 14157.

10 K. L. McCall, J. R. Jennings, H. Wang, A. Morandeira, L. M. Peter, J. R. Durrant, L. J. Yellowlees and N. Robertson, Eur. J. Inorg. Chem., 2011, 589.

11 J. Baldenebro-López, J. Castorena-González, N. FloresHolguín, J. Almaral-Sánchez and D. Glossman-Mitnik, Int. J. Mol. Sci., 2012, 13, 16005.

12 A. Hernandez Redondo, E. C. Constable and C. E. Housecroft, Chimia, 2009, 63, 205.

13 B. Bozic-Weber, E. C. Constable, C. E. Housecroft, P. Kopecky, M. Neuburger and J. A. Zampese, Dalton Trans., 2011, 40, 12584.

14 B. Bozic-Weber, S. Y. Brauchli, E. C. Constable, S. O. Fürer, C. E. Housecroft and I. A. Wright, Phys. Chem. Chem. Phys., 2013, 15, 4500.

15 P. Péchy, F. P. Rotzinger, Md. K. Nazeeruddin, O. Kohle, S. M. Zakeeruddin, R. Humphry-Baker and M. Grätzel, J. Chem. Soc., Chem. Commun., 1995, 65.

16 W. W. Brandt, F. P. Dwyer and E. D. Gyarfas, Chem. Rev., 1954, 545, 959.

17 A. K. Ichinaga, J. R. Kirchhoff, D. R. McMillin, C. O. Dietrich-Buchecker, P. A. Marnot and J.-P. Sauvage, Inorg. Chem., 1987, 26, 4290.

18 A. J. Pallenberg, K. S. Koenig and D. M. Barnhart, Inorg. Chem., 1995, 34, 2833.
19 M. K. Eggleston, D. R. McMillin, K. S. Koenig and A. J. Pallenberg, Inorg. Chem., 1997, 36, 172.

20 M. T. Miller, P. K. Gantzel and T. B. Karpishin, Inorg. Chem., 1998, 37, 2285.

21 P. Yang, X.-J. Yang and B. Wu, Eur. J. Inorg. Chem., 2009, 2951.

22 N. A. Gothard, M. W. Mara, J. Huang, J. M. Szarko, B. Rolczynski, J. V. Lockard and L. X. Chen, J. Phys. Chem. A, 2012, 116, 1984.

23 A. Hernández Redondo, PhD Thesis, University of Basel, 2009.

24 V. Penicaud, F. Odobel and B. Bujoli, Tetrahedron Lett., 1998, 39, 3689.

25 APEX2, version 2 User Manual, M86-E01078, Bruker Analytical X-ray Systems, Inc., Madison, WI, 2006.

26 G. M. Sheldrick, Acta Crystallogr., Sect. A: Fundam. Crystallogr., 2008, 64, 112.

27 I. J. Bruno, J. C. Cole, P. R. Edgington, M. K. Kessler, C. F. Macrae, P. McCabe, J. Pearson and R. Taylor, Acta Crystallogr., Sect. B: Struct. Sci., 2002, 58, 389.

28 C. F. Macrae, I. J. Bruno, J. A. Chisholm, P. R. Edgington, P. McCabe, E. Pidcock, L. Rodriguez-Monge, R. Taylor, J. van de Streek and P. A. Wood, J. Appl. Crystallogr., 2008, 41, 466.

29 S. Ito, T. N. Murakami, P. Comte, P. Liska, C. Grätzel, M. K. Nazeeruddin and M. Grätzel, Thin Solid Films, 2008, 516, 4613.

30 F. Krönhke, Synthesis, 1976, 1.

31 V. Penicaud, F. Odobel and B. Bujoli, Tetrahedron Lett., 1998, 39, 3689.

32 B. C. K. Chan and M. C. Baird, Inorg. Chim. Acta, 2004, 357, 2776.

33 S. Y. Brauchli, E. C. Constable and C. E. Housecroft, unpublished results.

34 P. J. Burke, D. R. McMillin and W. R. Robinson, Inorg. Chem., 1980, 19, 1211.

35 S. Itoh, N. Kishikawa, T. Suzuki and H. G. Takagi, Dalton Trans., 2005, 1066.

36 See for example: C. M. L. Vande Velde, M. Zeller and V. A. Azov, J. Mol. Struct., 2012, 1016, 109; M. Fourmigue and P. Batail, Chem. Rev., 2004, 104, 5379.

37 S. Kume, M. Murata, T. Ozeki and H. Nishihara, J. Am. Chem. Soc., 2005, 127, 490.

38 R. M. Williams, L. De Cola, F. Hartl, J.-J. Lagref, J.-M. Planeix, A. De Cian and M. W. Hosseini, Coord. Chem. Rev., 2002, 230, 253.

39 H. Akdas-Kilig, J.-P. Malval, F. Morlet-Savary, A. Singh, L. Toupet, I. Ledoux-Rak, J. Zyss and H. Le Bozec, Dyes Pigm., 2011, 92, 681.

40 F. F. de Biani, E. Grigiotti, F. Laschi, P. Zanello, A. Juris, L. Prodi, K. S. Chichak and N. R. Branda, Inorg. Chem., 2008, 47, 5425.

41 D. A. Bardwell, J. C. Jeffery, C. A. Otter and M. D. Ward, Polyhedron, 1996, 15, 191.

42 M. Cesario, C. O. Dietrich-Buchecker, J. Guilhem, C. Pascard and J.-P. Sauvage, J. Chem. Soc., Chem. Commun., 1985, 244. 
43 C. O. Dietrich-Buchecker, J. Guilhem, A. K. Khemiss, J.-P. Kintzinger, C. Pascard and J.-P. Sauvage, Angew. Chem., Int. Ed., 1987, 26, 661.

44 C. Dietrich-Buchecker, G. Rapenne, J.-P. Sauvage, A. De Cian and J. Fischer, Chem.-Eur. J., 1999, 5, 1432.

45 C. Dietrich-Buchecker, N. G. Hwang and J.-P. Sauvage, New J. Chem., 1999, 23, 911.

46 F. K. Klemens, C. E. A. Palmer, S. M. Rolland, P. E. Fanwick, D. R. McMillin and J.-P. Sauvage, New J. Chem., 1990, 14, 129.

47 J.-C. Chambron, J.-P. Sauvage, K. Mislow, A. De Cian and J. Fischer, Chem.-Eur. J., 2001, 7, 4085.

48 M. Geoffroy, M. Wermeille, C. O. Buchecker, J.-P. Sauvage and G. Bernardinelli, Inorg. Chim. Acta, 1990, 167, 157.
49 B. Wu, P. Yang, X. Huang, Y. Liu, X. Liu and C. Xia, Z. Anorg. Allg. Chem., 2006, 632, 684.

50 C. T. Cunningham, J. J. Moore, K. L. H. Cunningham, P. E. Fanwick and D. R. McMillin, Inorg. Chem, 2000, 39, 3638.

51 V. V. Pavlishchuk and A. W. Addison, Inorg. Chim. Acta, 2000, 298, 97.

52 H. Mohan and J. P. Mittal, J. Phys. Chem., 1995, 99, 6519.

53 Y. Jahng, J. Hazelrigg, D. Kimball, E. Riesgo, R. Wu and R. P. Thummel, Inorg. Chem., 1997, 36, 5390.

54 D. V. Scaltrito, D. W. Thompson, J. A. O'Callaghan and G. J. Meyer, Coord. Chem. Rev., 2000, 208, 243.

55 H. J. Snaith, Energy Environ. Sci., 2012, 5, 6513. 